\title{
ON OPTIMIZATION OF MANUFACTURING OF AN AMPLIFIER TO INCREASE DENSITY OF BIPOLAR TRANSISTOR FRAMEWORK THE AMPLIFIER
}

\author{
E.L. Pankratov and E.A. Bulaeva \\ Nizhny Novgorod State University, 23 Gagarin avenue, Nizhny Novgorod, 603950, \\ Russia
}

\begin{abstract}
In this paper we consider a possibility to increase density of bipolar heterotransistor framework an amplifier due to decreasing of their dimensions. The considered approach based on doping of required areas of heterostructure with specific configuration by diffusion or ion implantation. The doping finished by optimized annealing of dopant and/or radiation defects. Analysis of redistribution of dopant with account redistribution of radiation defects (after implantation of ions of dopant) for optimization of the above annealing have been done by using recently introduced analytical approach. The approach gives a possibility to analyze mass and heat transports in a heterostructure without crosslinking of solutions on interfaces between layers of the heterostructure with account nonlinearity of these transports and variation in time of their parameters.
\end{abstract}

\section{KEYWORDS}

Bipolar heterotransistor; increasing of density of transistor; an amplifier circuit

\section{INTRODUCTION}

In the present time an actual question is decreasing of dimensions of solid state electronic devices. To decrease the dimensions are could be increased density of elements of integrated circuits and decreased dimensions of these elements. To date, several methods to decrease dimensions of elements of integrated circuits have been developed. One of them is growth of thin films structures [1-5]. The second approach is diffusion or ion doping of required areas of samples or heterostructures and father laser or microwave annealing of dopant and/or radiation defects [6-8]. Using of the above approaches of annealing leads to generation of inhomogenous distribution of temperature and consequently to decreasing of dimensions of elements of integrated circuits. Another approach to change properties of doped materials is radiation processing $[9,10]$.

In the present paper we consider circuits of an amplifier [11] (see Fig. 1). Based on recently formulated recommendations to decrease dimensions of single transistors (both bipolar and fieldeffect) [12-17] we formulate recommendations to increase density of bipolar framework the considered circuits. We assume, that the considered circuits were manufactured framework the considered in Fig.1 heterostructure. The heterostructure consist of a substrate and an epitaxial layer. The epitaxial layer includes into itself several sections, manufactured by using other materials. These sections were doped by diffusion or by ion implantation to generate required types of conductivity $(n$ or $p$ ) in the required materials. Framework this paper we analyzed redistribution of dopants during annealing of these dopants and/or radiation defects to formulate conditions, which correspond to decreasing of the considered circuits. 


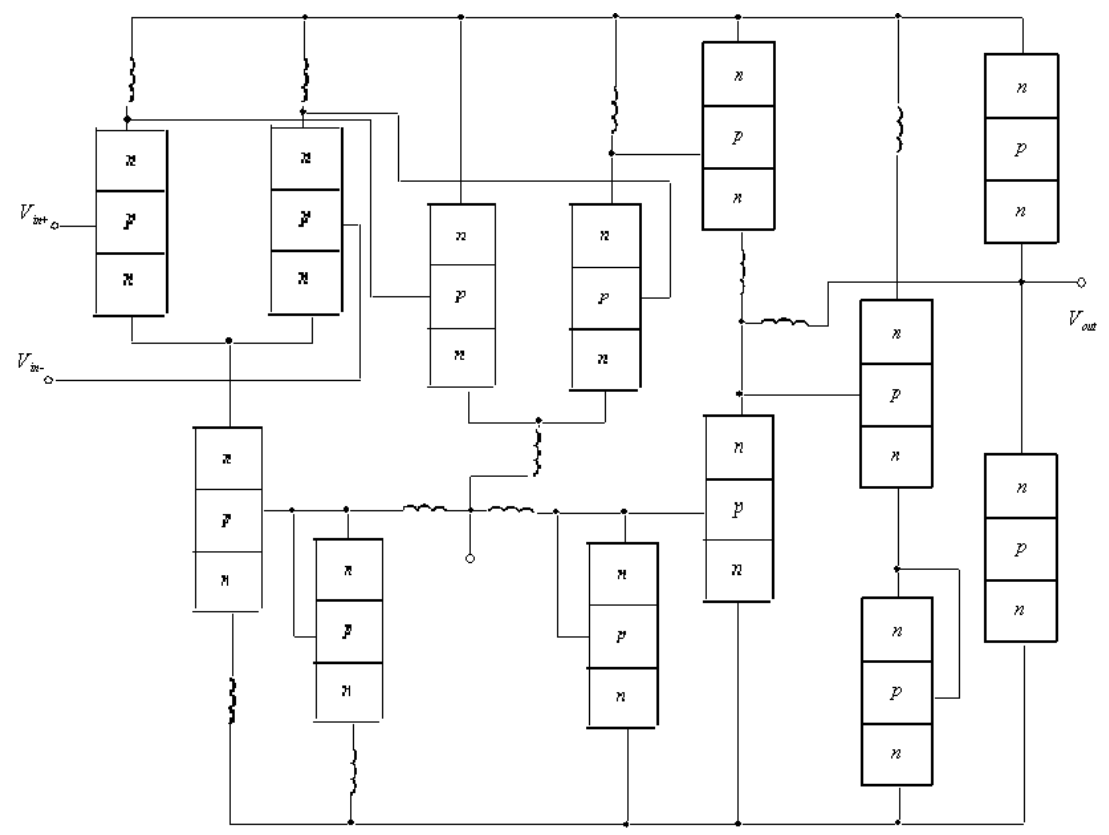

Fig. 1. Structure of inverter [11]. View from top on epitaxial layer

\section{Method OF SOLUTiON}

To solve our aims let us determine spatio-temporal distributions of concentrations of dopants. The required distributions we determined by solving the second Fick's law $[9,10,18,19]$

$$
\frac{\partial C(x, y, z, t)}{\partial t}=\frac{\partial}{\partial x}\left[D_{C} \frac{\partial C(x, y, z, t)}{\partial x}\right]+\frac{\partial}{\partial y}\left[D_{C} \frac{\partial C(x, y, z, t)}{\partial y}\right]+\frac{\partial}{\partial z}\left[D_{C} \frac{\partial C(x, y, z, t)}{\partial z}\right] .
$$

Boundary and initial conditions for the equations are

$$
\begin{gathered}
\left.\frac{\partial C(x, y, z, t)}{\partial x}\right|_{x=0}=0,\left.\frac{\partial C(x, y, z, t)}{\partial x}\right|_{x=L_{x}}=0,\left.\frac{\partial C(x, y, z, t)}{\partial y}\right|_{y=0}=0,\left.\frac{\partial C(x, y, z, t)}{\partial y}\right|_{x=L_{y}}=0, \\
\left.\frac{\partial C(x, y, z, t)}{\partial z}\right|_{z=0}=0,\left.\frac{\partial C(x, y, z, t)}{\partial z}\right|_{x=L_{z}}=0, C(x, y, z, 0)=f(x, y, z) .
\end{gathered}
$$

In the Eqs. (1) and (2) the function $C(x, y, z, t)$ describes the spatio-temporal distribution of concentration of dopant; the parameter $D_{C}$ is the dopant diffusion coefficient. Dopant diffusion coefficient will be changed with changing of materials of heterostructure, heating and cooling of heterostructure during annealing of dopant or radiation defects (with account Arrhenius law). Dependences of dopant diffusion coefficient on coordinate in heterostructure, temperature of annealing and concentrations of dopant and radiation defects could be written as $[10,18]$

$$
D_{C}=D_{L}(x, y, z, T)\left[1+\xi \frac{C^{\gamma}(x, y, z, t)}{P^{\gamma}(x, y, z, T)}\right]\left[1+\varsigma_{1} \frac{V(x, y, z, t)}{V^{*}}+\varsigma_{2} \frac{V^{2}(x, y, z, t)}{\left(V^{*}\right)^{2}}\right] .
$$


Here function $D_{L}(x, y, z, T)$ describes dependences of dopant diffusion coefficient on coordinate and temperature of annealing $T$. Function $P(x, y, z, T)$ describes the same dependences of the limit of solubility of dopant. The parameter $\gamma$ is integer and usually could be varying in the following interval $\gamma \in[1,3]$. The parameter describes quantity of charged defects, which interacting (in average) with each atom of dopant. More detailed information about concentrational dependence of dopant diffusion coefficient is presented in [18]. The function $V(x, y, z, t)$ describes distribution of concentration of radiation vacancies in space and time. The parameter $V^{*}$ describes the equilibrium distribution of concentration of vacancies. It should be noted, that diffusion type of doping gives a possibility to obtain doped materials without radiation defects. In this situation $\zeta_{1}=\zeta_{2}=0$. We determine spatio-temporal distributions of concentrations of radiation defects by solving the following system of equations $[10,13]$

$$
\begin{aligned}
& \frac{\partial I(x, y, z, t)}{\partial t}=\frac{\partial}{\partial x}\left[D_{I}(x, y, z, T) \frac{\partial I(x, y, z, t)}{\partial x}\right]+\frac{\partial}{\partial y}\left[D_{I}(x, y, z, T) \frac{\partial I(x, y, z, t)}{\partial y}\right]-k_{I, I}(x, y, z, T) \times \\
& \quad \times I^{2}(x, y, z, t)+\frac{\partial}{\partial z}\left[D_{I}(x, y, z, T) \frac{\partial I(x, y, z, t)}{\partial z}\right]-k_{I, V}(x, y, z, T) I(x, y, z, t) V(x, y, z, t) \quad \text { (4) } \\
& \frac{\partial V(x, y, z, t)}{\partial t}=\frac{\partial}{\partial x}\left[D_{V}(x, y, z, T) \frac{\partial V(x, y, z, t)}{\partial x}\right]+\frac{\partial}{\partial y}\left[D_{V}(x, y, z, T) \frac{\partial V(x, y, z, t)}{\partial y}\right]-k_{V, V}(x, y, z, T) \times \\
& \quad \times V^{2}(x, y, z, t)+\frac{\partial}{\partial z}\left[D_{V}(x, y, z, T) \frac{\partial V(x, y, z, t)}{\partial z}\right]-k_{I, V}(x, y, z, T) I(x, y, z, t) V(x, y, z, t) .
\end{aligned}
$$

Boundary and initial conditions for these equations are

$$
\begin{gathered}
\left.\frac{\partial \rho(x, y, z, t)}{\partial x}\right|_{x=0}=0,\left.\frac{\partial \rho(x, y, z, t)}{\partial x}\right|_{x=L_{x}}=0,\left.\frac{\partial \rho(x, y, z, t)}{\partial y}\right|_{y=0}=0,\left.\frac{\partial \rho(x, y, z, t)}{\partial y}\right|_{y=L_{y}}=0, \\
\left.\frac{\partial \rho(x, y, z, t)}{\partial z}\right|_{z=0}=0,\left.\frac{\partial \rho(x, y, z, t)}{\partial z}\right|_{z=L_{z}}=0, \rho(x, y, z, 0)=f_{\rho}(x, y, z) .
\end{gathered}
$$

Here $\rho=I, V$. The function $I(x, y, z, t)$ describes the distribution of concentration of radiation interstitials in space and time. The functions $D_{\rho}(x, y, z, T)$ describe dependences of the diffusion coefficients of point radiation defects on coordinate and temperature. The quadric on concentrations terms of Eqs. (4) describes generation divacancies and diinterstitials. The function $k_{I, V}(x, y, z, T)$ describes dependence of the parameter of recombination of point radiation defects on coordinate and temperature. The function $k_{I, I}(x, y, z, T)$ and $k_{V, V}(x, y, z, T)$ describes dependences of the parameters of generation of simplest complexes of point radiation defects on coordinate and temperature.

Now let us calculate distributions of concentrations of divacancies $\Phi_{V}(x, y, z, t)$ and diinterstitials $\Phi_{I}(x, y, z, t)$ in space and time by solving the following system of equations $[10,19]$

$$
\begin{aligned}
& \frac{\partial \Phi_{I}(x, y, z, t)}{\partial t}=\frac{\partial}{\partial x}\left[D_{\Phi I}(x, y, z, T) \frac{\partial \Phi_{I}(x, y, z, t)}{\partial x}\right]+\frac{\partial}{\partial y}\left[D_{\Phi I}(x, y, z, T) \frac{\partial \Phi_{I}(x, y, z, t)}{\partial y}\right]+ \\
& +\frac{\partial}{\partial z}\left[D_{\Phi I}(x, y, z, T) \frac{\partial \Phi_{I}(x, y, z, t)}{\partial z}\right]+k_{I, I}(x, y, z, T) I^{2}(x, y, z, t)-k_{I}(x, y, z, T) I(x, y, z, t)
\end{aligned}
$$




$$
\begin{aligned}
& \frac{\partial \Phi_{V}(x, y, z, t)}{\partial t}=\frac{\partial}{\partial x}\left[D_{\Phi V}(x, y, z, T) \frac{\partial \Phi_{V}(x, y, z, t)}{\partial x}\right]+\frac{\partial}{\partial y}\left[D_{\Phi V}(x, y, z, T) \frac{\partial \Phi_{V}(x, y, z, t)}{\partial y}\right]+ \\
& +\frac{\partial}{\partial z}\left[D_{\Phi V}(x, y, z, T) \frac{\partial \Phi_{V}(x, y, z, t)}{\partial z}\right]+k_{V, V}(x, y, z, T) V^{2}(x, y, z, t)-k_{V}(x, y, z, T) V(x, y, z, t) .
\end{aligned}
$$

Boundary and initial conditions for these equations are

$$
\begin{gathered}
\left.\frac{\partial \Phi_{\rho}(x, y, z, t)}{\partial x}\right|_{x=0}=0,\left.\frac{\partial \Phi_{\rho}(x, y, z, t)}{\partial x}\right|_{x=L_{x}}=0,\left.\frac{\partial \Phi_{\rho}(x, y, z, t)}{\partial y}\right|_{y=0}=0,\left.\frac{\partial \Phi_{\rho}(x, y, z, t)}{\partial y}\right|_{y=L_{y}}=0 \\
\left.\frac{\partial \Phi_{\rho}(x, y, z, t)}{\partial z}\right|_{z=0}=0,\left.\frac{\partial \Phi_{\rho}(x, y, z, t)}{\partial z}\right|_{z=L_{z}}=0, \Phi_{I}(x, y, z, 0)=f_{\Phi I}(x, y, z), \Phi_{V}(x, y, z, 0)=f_{\Phi V}(x, y, z) .(7)
\end{gathered}
$$

The functions $D_{\phi \rho}(x, y, z, T)$ describe dependences of the diffusion coefficients of the above complexes of radiation defects on coordinate and temperature. The functions $k_{I}(x, y, z, T)$ and $k_{V}(x, y, z$, $T)$ describe the parameters of decay of these complexes on coordinate and temperature.

We calculate distributions of concentrations of point radiation defects in space and time by using recently elaborated approach $[12,13]$. To use the approach let us transform dependences of diffusion coefficients of point defects in space and time to the following form: $D_{\rho}(x, y, z, T)=D_{0 \rho}\left[1+\varepsilon_{\rho}\right.$ $\left.g_{\rho}(x, y, z, T)\right]$, where $D_{0 \rho}$ are the average values of diffusion coefficients, $0 \leq \varepsilon_{\rho}<1,\left|g_{\rho}(x, y, z, T)\right| \leq 1, \rho$ $=I, V$. Let us also transform dependences of another parameters to the similar form: $k_{I, V}(x, y, z, T)=$ $k_{0 I, V}\left[1+\varepsilon_{I, V} g_{I, V}(x, y, z, T)\right], \quad k_{I, I}(x, y, z, T)=k_{0 I I I}\left[1+\varepsilon_{I, I} \quad g_{I, I}(x, y, z, T)\right]$ and $k_{V, V}(x, y, z, T)=k_{0 V, V}\left[1+\varepsilon_{V, V}\right.$ $\left.g_{V, V}(x, y, z, T)\right]$, where $k_{0 \rho 1, \rho 2}$ are the their average values, $0 \leq \varepsilon_{I, V}<1,0 \leq \varepsilon_{I, I}<1,0 \leq \varepsilon_{V, V}<1$, I $g_{I, V}(x, y, z, T)|\leq 1,| g_{I, I}(x, y, z, T)|\leq 1,| g_{V, V}(x, y, z, T) \mid \leq 1$. Let us introduce the following dimensionless variables: $\tilde{I}(x, y, z, t)=I(x, y, z, t) / I^{*}, \chi=x / L_{x}, \eta=y / L_{y}, \phi=z / L_{z}, \tilde{V}(x, y, z, t)=V(x, y, z, t) / V^{*}$, $\vartheta=\sqrt{D_{0 I} D_{0 V}} t / L^{2}, \omega=L^{2} k_{0 I, V} / \sqrt{D_{0 I} D_{0 V}}, \Omega_{\rho}=L^{2} k_{0 \rho, \rho} / \sqrt{D_{0 I} D_{0 V}}$. The introduction leads to transformation of Eqs.(4) and conditions (5) to the following form

$$
\begin{gathered}
\frac{\partial \tilde{I}(\chi, \eta, \phi, \vartheta)}{\partial \vartheta}=\frac{D_{0 I}}{\sqrt{D_{0 I} D_{0 V}}} \frac{\partial}{\partial \chi}\left\{\left[1+\varepsilon_{I} g_{I}(\chi, \eta, \phi, T)\right] \frac{\partial \tilde{I}(\chi, \eta, \phi, \vartheta)}{\partial \chi}\right\}+\frac{\partial}{\partial \eta}\left\{1+\varepsilon_{I} g_{I}(\chi, \eta, \phi, T)\right] \times \\
\left.\times \frac{\partial \tilde{I}(\chi, \eta, \phi, \vartheta)}{\partial \eta}\right\} \frac{D_{0 I}}{\sqrt{D_{0 I} D_{0 V}}}+\frac{D_{0 I}}{\sqrt{D_{0 I} D_{0 V}}} \frac{\partial}{\partial \phi}\left\{\left[1+\varepsilon_{I} g_{I}(\chi, \eta, \phi, T)\right] \frac{\partial \tilde{I}(\chi, \eta, \phi, \vartheta)}{\partial \phi}\right\}-\tilde{I}(\chi, \eta, \phi, \vartheta) \times \\
\quad \times \omega\left[1+\varepsilon_{I, V} g_{I, V}(\chi, \eta, \phi, T)\right] \tilde{V}(\chi, \eta, \phi, \vartheta)-\Omega_{I}\left[1+\varepsilon_{I, I} g_{I, I}(\chi, \eta, \phi, T)\right] \tilde{I}^{2}(\chi, \eta, \phi, \vartheta) \\
\begin{array}{c}
\frac{\partial \tilde{V}(\chi, \eta, \phi, \vartheta)}{\partial \vartheta}=\frac{D_{0 V}}{\sqrt{D_{0 I} D_{0 V}}} \frac{\partial}{\partial \chi}\left\{\left[1+\varepsilon_{V} g_{V}(\chi, \eta, \phi, T)\right] \frac{\partial \tilde{V}(\chi, \eta, \phi, \vartheta)}{\partial \chi}\right\}+\frac{\partial}{\partial \eta}\left\{1+\varepsilon_{V} g_{V}(\chi, \eta, \phi, T)\right] \times \\
\left.\times \frac{\partial \tilde{V}(\chi, \eta, \phi, \vartheta)}{\partial \eta}\right\} \frac{D_{0 V}}{\sqrt{D_{0 I} D_{0 V}}}+\frac{D_{0 V}}{\sqrt{D_{0 I} D_{0 V}}} \frac{\partial}{\partial \phi}\left\{\left[1+\varepsilon_{V} g_{V}(\chi, \eta, \phi, T)\right] \frac{\partial \tilde{V}(\chi, \eta, \phi, \vartheta)}{\partial \phi}\right\}-\tilde{I}(\chi, \eta, \phi, \vartheta) \times \\
\quad \times \omega\left[1+\varepsilon_{I, V} g_{I, V}(\chi, \eta, \phi, T)\right] \tilde{V}(\chi, \eta, \phi, \vartheta)-\Omega_{V}\left[1+\varepsilon_{V, V} g_{V, V}(\chi, \eta, \phi, T)\right] \tilde{V}^{2}(\chi, \eta, \phi, \vartheta) \\
\left.\frac{\partial \tilde{\rho}(\chi, \eta, \phi, \vartheta)}{\partial \chi}\right|_{\chi=0}=0,\left.\frac{\partial \tilde{\rho}(\chi, \eta, \phi, \vartheta)}{\partial \chi}\right|_{\chi=1}=0,\left.\frac{\partial \tilde{\rho}(\chi, \eta, \phi, \vartheta)}{\partial \eta}\right|_{\eta=0}=0,\left.\frac{\partial \tilde{\rho}(\chi, \eta, \phi, \vartheta)}{\partial \eta}\right|_{\eta=1}=0,
\end{array}
\end{gathered}
$$




$$
\left.\frac{\partial \tilde{\rho}(\chi, \eta, \phi, \vartheta)}{\partial \phi}\right|_{\phi=0}=0,\left.\frac{\partial \tilde{\rho}(\chi, \eta, \phi, \vartheta)}{\partial \phi}\right|_{\phi=1}=0, \tilde{\rho}(\chi, \eta, \phi, \vartheta)=\frac{f_{\rho}(\chi, \eta, \phi, \vartheta)}{\rho^{*}} .
$$

Let us determine solutions of Eqs.(8) as the following power series [12,13]

$$
\tilde{\rho}(\chi, \eta, \phi, \vartheta)=\sum_{i=0}^{\infty} \varepsilon_{\rho}^{i} \sum_{j=0}^{\infty} \omega^{j} \sum_{k=0}^{\infty} \Omega_{\rho}^{k} \tilde{\rho}_{i j k}(\chi, \eta, \phi, \vartheta)
$$

After substitution of the series (10) into Eqs.(8) and conditions (9) we obtain equations for initial-order approximations of concentration of point defects $\tilde{I}_{000}(\chi, \eta, \phi, \vartheta)$ and $\tilde{V}_{000}(\chi, \eta, \phi, \vartheta)$, corrections $\tilde{I}_{i j k}(\chi, \eta, \phi, \vartheta)$ and $\tilde{V}_{i j k}(\chi, \eta, \phi, \vartheta)$ and conditions for them for all $i \geq 1, j \geq 1, k \geq 1$. The equations are presented in the Appendix. We calculate solutions of the equations by standard Fourier approach $[20,21]$. The solutions are presented in the Appendix.

Farther we determine spatio-temporal distributions of concentrations of simplest complexes of point radiation defects. To determine the distributions we transform approximations of diffusion coefficients in the following form: $D_{\Phi \rho}(x, y, z, T)=D_{0 \Phi \rho}\left[1+\varepsilon_{\Phi \rho} g_{\Phi \rho}(x, y, z, T)\right]$, where $D_{0 \Phi \rho}$ are the average values of diffusion coefficients. In this situation the Eqs.(6) could be written as

$$
\begin{aligned}
& \frac{\partial \Phi_{I}(x, y, z, t)}{\partial t}=D_{0 \Phi I} \frac{\partial}{\partial x}\left\{\left[1+\varepsilon_{\Phi I} g_{\Phi I}(x, y, z, T)\right] \frac{\partial \Phi_{I}(x, y, z, t)}{\partial x}\right\}+k_{I, I}(x, y, z, T) I^{2}(x, y, z, t)+ \\
& +D_{0 \Phi I} \frac{\partial}{\partial y}\left\{\left[1+\varepsilon_{\Phi I} g_{\Phi I}(x, y, z, T)\right] \frac{\partial \Phi_{I}(x, y, z, t)}{\partial y}\right\}+D_{0 \Phi I} \frac{\partial}{\partial z}\left\{\left[1+\varepsilon_{\Phi I} g_{\Phi l}(x, y, z, T)\right] \frac{\partial \Phi_{I}(x, y, z, t)}{\partial z}\right\}- \\
& -k_{I}(x, y, z, T) I(x, y, z, t) \\
& \frac{\partial \Phi_{V}(x, y, z, t)}{\partial t}=D_{0 \Phi V} \frac{\partial}{\partial x}\left\{\left[1+\varepsilon_{\Phi V} g_{\Phi V}(x, y, z, T)\right] \frac{\partial \Phi_{V}(x, y, z, t)}{\partial x}\right\}+k_{I, I}(x, y, z, T) I^{2}(x, y, z, t)+ \\
& +D_{0 \Phi V} \frac{\partial}{\partial y}\left\{\left[1+\varepsilon_{\Phi V} g_{\Phi V}(x, y, z, T)\right] \frac{\partial \Phi_{V}(x, y, z, t)}{\partial y}\right\}+D_{0 \Phi V} \frac{\partial}{\partial z}\left\{\left[1+\varepsilon_{\Phi V} g_{\Phi V}(x, y, z, T)\right] \frac{\partial \Phi_{V}(x, y, z, t)}{\partial z}\right\}- \\
& -k_{I}(x, y, z, T) I(x, y, z, t) .
\end{aligned}
$$

We determine spatio-temporal distributions of concentrations of complexes of radiation defects as the following power series

$$
\Phi_{\rho}(x, y, z, t)=\sum_{i=0}^{\infty} \varepsilon_{\Phi \rho}^{i} \Phi_{\rho i}(x, y, z, t) .
$$

Equations for the functions $\Phi_{\rho_{i}}(x, y, z, t)$ and conditions for them could be obtained by substitution of the series (11) into Eqs.(6) and appropriate boundary and initial conditions. We present the equations and conditions in the Appendix. Solutions of the equations have been calculated by standard approaches $[20,21]$ and presented in the Appendix.

Now we calculate distribution of concentration of dopant in space and time by using the same approach, which was used for calculation the same distributions of another concentrations. To use the approach we transform spatio-temperature approximation of dopant diffusion coefficient to the form: $D_{L}(x, y, z, T)=D_{0 L}\left[1+\varepsilon_{L} g_{L}(x, y, z, T)\right]$, where $D_{0 L}$ is the average value of dopant diffusion coefficient, $0 \leq \varepsilon_{L}<1,\left|g_{L}(x, y, z, T)\right| \leq 1$. Now we solve the Eq.(1) as the following power series 


$$
C(x, y, z, t)=\sum_{i=0}^{\infty} \varepsilon_{L}^{i} \sum_{j=1}^{\infty} \xi^{j} C_{i j}(x, y, z, t)
$$

The equations for the functions $C_{i j}(x, y, z, t)$ and conditions for them have been obtained by substitution of the series into Eq.(1) and conditions (2). We presented the equations and conditions for them in the Appendix. We solve the equations by standard Fourier approach [20,21]. The solutions have been presented in the Appendix.

We analyzed distributions of concentrations of dopant and radiation defects in space and time analytically by using the second-order approximations on all parameters, which have been used in appropriate series. The approximations are usually enough good approximations to make qualitative analysis and to obtain quantitative results. We check all results of analytical modeling by comparison with results of numerical simulation.

\section{DISCUSSION}

In this section based on recently calculated relations we analyzed redistribution of dopant with account redistribution of radiation defects. These relations give us possibility to obtain spatial distributions of concentration of dopant. Typical spatial distributions of concentration of dopant in directions, which is perpendicular to channel, are presented in Fig. 2. Curve 1 of this paper is a typical distribution of concentration of dopant in directions of channel. The figure shows, that presents of interface between layers of heterostructure gives us possibility to obtain more compact and more homogenous distribution of concentration of dopant in direction, which is perpendicular to the interface. However in this situation one shall to optimize annealing time. Reason of the optimization is following. If annealing time is small, dopant can not achieves the interface. If annealing time is large, dopant will diffuse into another layers of heterostructure too deep. We calculate optimal value of annealing time by using recently introduced criterion [12-17]. Framework the criterion we approximate real distribution of concentration of dopant by ideal step-wise function $\psi(x, y, z)$. Farther the required optimal value of dopant concentration by minimization of the following mean-squared error

$$
U=\frac{1}{L_{x} L_{y} L_{z}} \int_{0}^{L_{x}} \int_{0}^{L_{y} L_{z}} \int_{0}[C(x, y, z, \Theta)-\psi(x, y, z)] d z d y d x
$$

Dependences of optimal value of annealing time are presented on Figs. 3. Optimal values of annealing time of implanted dopant should be smaller in comparison with the same annealing time of infused dopant. Reason of the difference is necessity to anneal radiation defects before annealing of dopant. 


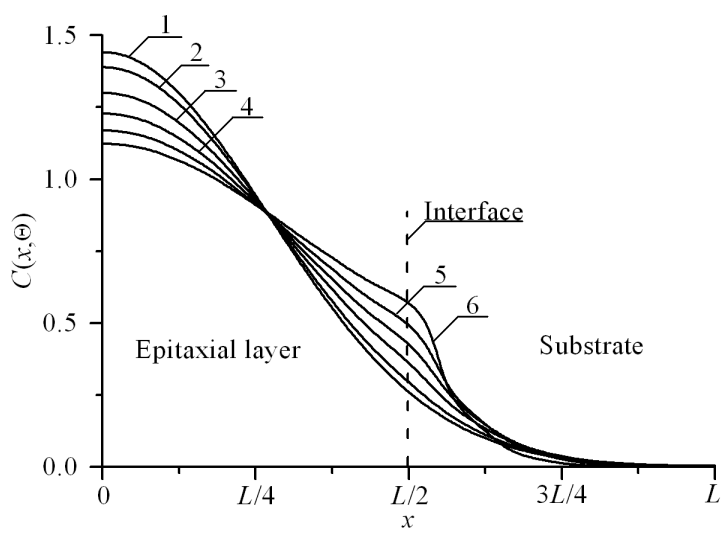

Fig.2. The infused dopant concentration distributions. The considered direction is perpendicular to interface between epitaxial layer and substrate. Increasing of number of distributions corresponds to increasing of difference between values of dopant diffusion coefficient in layers of heterostructure. The distributions have been calculated under condition, when value of dopant diffusion coefficient in epitaxial layer is larger, than value of dopant diffusion coefficient in substrate

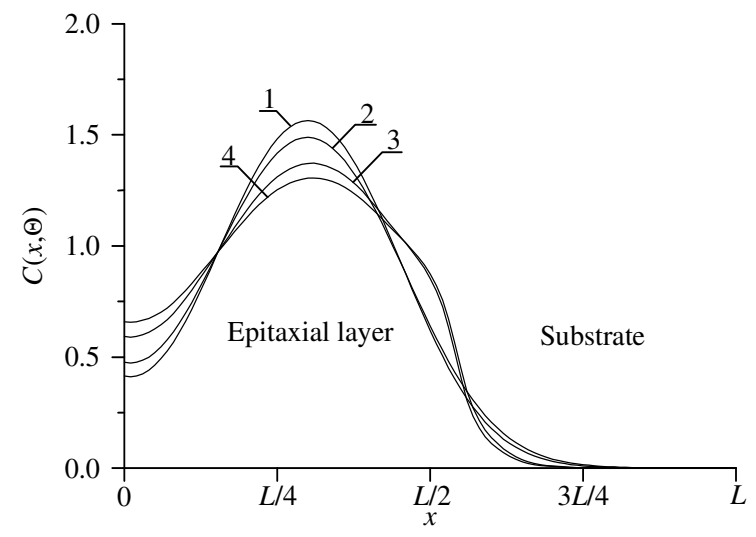

Fig.3. Fig.2. The implanted dopant concentration distributions. The considered direction is perpendicular to interface between epitaxial layer and substrate. Curves 1 and 3 corresponds to annealing time $\Theta=$ $0.0048\left(L_{x}{ }^{2}+L_{y}{ }^{2}+L_{z}{ }^{2}\right) / D_{0}$. Curves 2 and 4 corresponds to annealing time $\Theta=0.0057\left(L_{x}{ }^{2}+L_{y}{ }^{2}+L_{z}{ }^{2}\right) / D_{0}$. Curves 1 and 2 have been calculated for homogenous sample. Curves 3 and 4 have been calculated for heterostructure under condition, when value of dopant diffusion coefficient in epitaxial layer is larger, than value of dopant diffusion coefficient in substrate

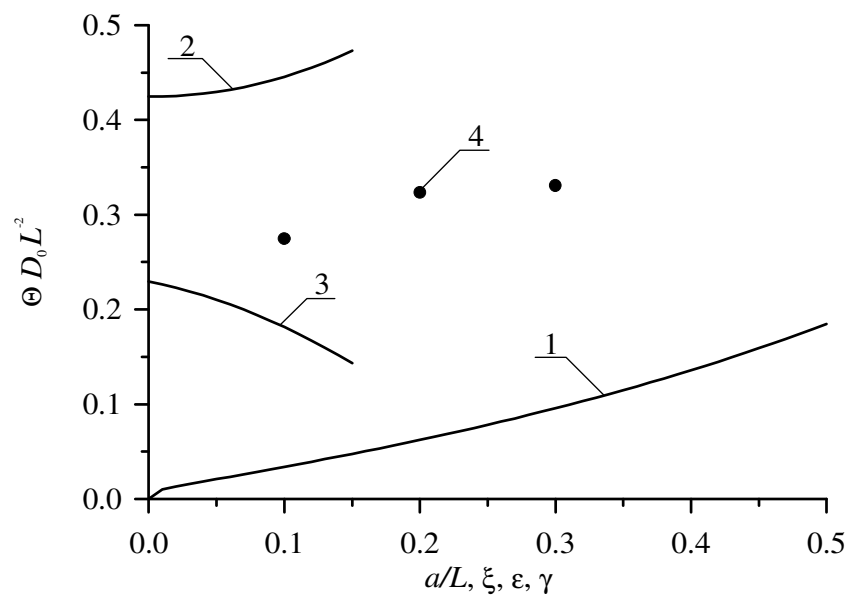


Fig.3a. Optimal annealing time of infused dopant as dependences of several parameters. Curve 1 is the dependence of the considered annealing time on dimensionless thickness of epitaxial layer $a / L$ and $\xi=\gamma=0$ for equal to each other values of dopant diffusion coefficient in all parts of heterostructure. Curve 2 is the dependence of the considered annealing time on the parameter $\varepsilon$ for $a / L=1 / 2$ and $\xi=\gamma=0$. Curve 3 is the dependence of the considered annealing time on the parameter $\xi$ for $a / L=1 / 2$ and $\varepsilon=\gamma=0$. Curve 4 is the dependence of the considered annealing time on parameter $\gamma$ for $a / L=1 / 2$ and $\varepsilon=\xi=0$

\section{CONCLUSIONS}

In this paper we consider a possibility to increase density of elements in circuit of an amplifier based on bipolar heterotransistors. Several conditions to increase the density have been formulated. Analysis of redistribution of dopant with account redistribution of radiation defects (after implantation of ions of dopant) for optimization of the above annealing have been done by using recently introduced analytical approach. The approach gives a possibility to analyze mass and heat transports in a heterostructure without crosslinking of solutions on interfaces between layers of the heterostructure with account nonlinearity of these transports and variation in time of their parameters.

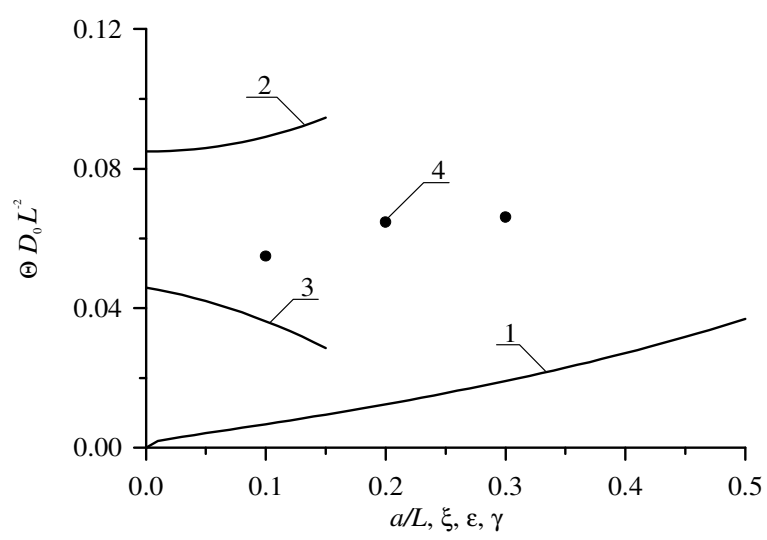

Fig.3a. Optimal annealing time of implanted dopant as dependences of several parameters. Curve 1 is the dependence of the considered annealing time on dimensionless thickness of epitaxial layer $a / L$ and $\xi=\gamma=0$ for equal to each other values of dopant diffusion coefficient in all parts of heterostructure. Curve 2 is the dependence of the considered annealing time on the parameter $\varepsilon$ for $a / L=1 / 2$ and $\xi=\gamma=0$. Curve 3 is the dependence of the considered annealing time on the parameter $\xi$ for $a / L=1 / 2$ and $\varepsilon=\gamma=0$. Curve 4 is the dependence of the considered annealing time on parameter $\gamma$ for $a / L=1 / 2$ and $\varepsilon=\xi=0$

\section{ACKNOWLEDGEMENTS}

This work is supported by the agreement of August 27, 2013 № 02.B.49.21.0003 between The Ministry of education and science of the Russian Federation and Lobachevsky State University of Nizhni Novgorod.

\section{REFERENCES}

1. G. Volovich. Modern chips UM3Ch class D manufactured by firm MPS. Modern Electronics. Issue 2. P. 10-17 (2006).

2. A. Kerentsev, V. Lanin. Constructive-technological features of MOSFET-transistors. Power Electronics. Issue 1. P. 34-38 (2008).

3. O.A. Ageev, A.E. Belyaev, N.S. Boltovets, V.N. Ivanov, R.V. Konakova, Ya.Ya. Kudryk, P.M. 
Lytvyn, V.V. Milenin, A.V. Sachenko. Influence of displacement of the electron-hole equilibrium on the process of transition metals diffusion in GaAs. Semiconductors. Vol. 43 (7). P. 897-903 (2009).

4. N.I. Volokobinskaya, I.N. Komarov, T.V. Matioukhina, V.I. Rechetniko, A.A. Rush, I.V. Falina, A.S. Yastrebov. Investigation of technological processes of manufacturing of the bipolar power high-voltage transistors with a grid of inclusions in the collector region. Semiconductors. Vol. 35 (8). P. 1013-1017 (2001).

5. A. Subramaniam, K. D. Cantley, E.M. Vogel. Active and Passive Electronic Components. Vol. 2013, ID 525017 (2013).

6. K.K. Ong, K.L. Pey, P.S. Lee, A.T.S. Wee, X.C. Wang, Y.F. Chong. Dopant distribution in the recrystallization transient at the maximum melt depth induced by laser annealing. Appl. Phys. Lett. 89 (17), 172111-172114 (2006).

7. H.T. Wang, L.S. Tan, E. F. Chor. Pulsed laser annealing of Be-implanted GaN. J. Appl. Phys. 98 (9), 094901-094905 (2006).

8. Yu.V. Bykov, A.G. Yeremeev, N.A. Zharova, I.V. Plotnikov, K.I. Rybakov, M.N. Drozdov, Yu.N. Drozdov, V.D. Skupov. Diffusion processes in semiconductor structures during microwave annealing. Radiophysics and Quantum Electronics. Vol. 43 (3). P. 836-843 (2003).

9. V.V. Kozlivsky. Modification of semiconductors by proton beams (Nauka, Sant-Peterburg, 2003, in Russian).

10. V.L. Vinetskiy, G.A. Kholodar', Radiative physics of semiconductors. ("Naukova Dumka", Kiev, 1979, in Russian).

11. S. Kargarrazi, L. Lanni, C.-M. Zetterling. Solid-State Electronics. Vol. 116. P. 33-37 (2016).

12. E.L. Pankratov, E.A. Bulaeva. Increasing of sharpness of diffusion-junction heterorectifier by using radiation processing. Int. J. Nanoscience. Vol. 11 (5). P. 1250028-1--1250028-8 (2012).

13. E.L. Pankratov, E.A. Bulaeva. About some ways to decrease quantity of defects in materials for solid state electronic devices and diagnostics of their realization. Reviews in Theoretical Science. Vol. 3 (2). P. 177-215 (2015).

14. E.L. Pankratov, E.A. Bulaeva. An approach to decrease dimentions of logical elements based on bipolar transistor. Int. J. Comp. Sci. Appl. Vol. 5 (4). P. 1-18 (2015).

15. E.L. Pankratov, E.A. Bulaeva. On approach to decrease dimensions of element OR manufactured by using field-effect heterotransistor. Nano Science and Nano Technology: An Indian Journal. Vol. 9 (4). P. 43-60 (2015).

16. E.L. Pankratov, E.A. Bulaeva. Optimization of manufacturing of emitter-coupled logic to decrease surface of chip. Int. J. Mod. Phys. B. Vol. 29 (5). P. 1550023-1-1550023-12 (2015).

17. E.L. Pankratov, E.A. Bulaeva. Decreasing of mechanical stress in a semiconductor heterostructure by radiation processing. J. Comp. Theor. Nanoscience. Vol. 11 (1). P. 91-101 (2014).

18. Z.Yu. Gotra. Technology of microelectronic devices (Radio and communication, Moscow, 1991).

19. P.M. Fahey, P.B. Griffin, J.D. Plummer. Diffusion and point defects in silicon. Rev. Mod. Phys. 1989. V. 61. № 2. P. 289-388.

20. A.N. Tikhonov, A.A. Samarskii. The mathematical physics equations (Moscow, Nauka 1972) (in Russian).

21. H.S. Carslaw, J.C. Jaeger. Conduction of heat in solids (Oxford University Press, 1964).

\section{Authors:}

Pankratov Evgeny Leonidovich is a Full Doctor of Science, an Associate Professor of Nizhny Novgorod State University. He has 186 published papers in area of his researches.

Bulaeva Elena Alexeevna is a PhD student of Nizhny Novgorod State University. She has 134 published papers in area of her researches.

\section{APPENDIX}

Equations for the functions $\tilde{I}_{i j k}(\chi, \eta, \phi, \vartheta)$ and $\tilde{V}_{i j k}(\chi, \eta, \phi, \vartheta), i \geq 0, j \geq 0, k \geq 0$ and conditions for them 


$$
\begin{aligned}
& \frac{\partial \tilde{I}_{000}(\chi, \eta, \phi, \vartheta)}{\partial \vartheta}=\sqrt{\frac{D_{0 I}}{D_{0 V}}} \frac{\partial^{2} \tilde{I}_{000}(\chi, \eta, \phi, \vartheta)}{\partial \chi^{2}}+\sqrt{\frac{D_{0 I}}{D_{0 V}}} \frac{\partial^{2} \tilde{I}_{000}(\chi, \eta, \phi, \vartheta)}{\partial \eta^{2}}+\sqrt{\frac{D_{0 I}}{D_{0 V}}} \frac{\partial^{2} \tilde{I}_{000}(\chi, \eta, \phi, \vartheta)}{\partial \phi^{2}} \\
& \frac{\partial \tilde{V}_{000}(\chi, \eta, \phi, \vartheta)}{\partial \vartheta}=\sqrt{\frac{D_{0 V}}{D_{0 I}}} \frac{\partial^{2} \tilde{V}_{000}(\chi, \eta, \phi, \vartheta)}{\partial \chi^{2}}+\sqrt{\frac{D_{0 V}}{D_{0 I}}} \frac{\partial^{2} \tilde{V}_{000}(\chi, \eta, \phi, \vartheta)}{\partial \eta^{2}}+\sqrt{\frac{D_{0 V}}{D_{0 I}}} \frac{\partial^{2} \tilde{V}_{000}(\chi, \eta, \phi, \vartheta)}{\partial \phi^{2}} ; \\
& \frac{\partial \tilde{I}_{i 00}(\chi, \vartheta)}{\partial \vartheta}=\sqrt{\frac{D_{0 I}}{D_{0 V}}}\left[\frac{\partial^{2} \tilde{I}_{i 00}(\chi, \eta, \phi, \vartheta)}{\partial \chi^{2}}+\frac{\partial^{2} \tilde{I}_{i 00}(\chi, \eta, \phi, \vartheta)}{\partial \eta^{2}}+\frac{\partial^{2} \tilde{I}_{i 00}(\chi, \eta, \phi, \vartheta)}{\partial \phi^{2}}\right]+\frac{\partial}{\partial \chi}\left[g_{I}(\chi, \eta, \phi, T) \times\right. \\
& \left.\times \frac{\partial \tilde{I}_{i-100}(\chi, \eta, \phi, \vartheta)}{\partial \chi}\right] \sqrt{\frac{D_{0 I}}{D_{0 V}}}+\sqrt{\frac{D_{0 I}}{D_{0 V}}} \frac{\partial}{\partial \eta}\left[g_{I}(\chi, \eta, \phi, T) \frac{\partial \tilde{I}_{i-100}(\chi, \eta, \phi, \vartheta)}{\partial \eta}\right]+\frac{\partial}{\partial \phi}\left[g_{I}(\chi, \eta, \phi, T) \times\right. \\
& \left.\times \frac{\partial \tilde{I}_{i-100}(\chi, \eta, \phi, \vartheta)}{\partial \phi}\right] \sqrt{\frac{D_{0 I}}{D_{0 V}}}, i \geq 1, \\
& \frac{\partial \tilde{V}_{i 00}(\chi, \vartheta)}{\partial \vartheta}=\sqrt{\frac{D_{0 V}}{D_{0 I}}}\left[\frac{\partial^{2} \tilde{V}_{i 00}(\chi, \eta, \phi, \vartheta)}{\partial \chi^{2}}+\frac{\partial^{2} \tilde{V}_{i 00}(\chi, \eta, \phi, \vartheta)}{\partial \eta^{2}}+\frac{\partial^{2} \tilde{V}_{i 00}(\chi, \eta, \phi, \vartheta)}{\partial \phi^{2}}\right]+\frac{\partial}{\partial \chi}\left[g_{V}(\chi, \eta, \phi, T) \times\right. \\
& \left.\times \frac{\partial \tilde{V}_{i-100}(\chi, \eta, \phi, \vartheta)}{\partial \chi}\right] \sqrt{\frac{D_{0 V}}{D_{0 I}}}+\sqrt{\frac{D_{0 V}}{D_{0 I}}} \frac{\partial}{\partial \eta}\left[g_{V}(\chi, \eta, \phi, T) \frac{\partial \tilde{V}_{i-100}(\chi, \eta, \phi, \vartheta)}{\partial \eta}\right]+\frac{\partial}{\partial \phi}\left[g_{V}(\chi, \eta, \phi, T) \times\right. \\
& \left.\times \frac{\partial \tilde{V}_{i-100}(\chi, \eta, \phi, \vartheta)}{\partial \phi}\right] \sqrt{\frac{D_{0 V}}{D_{0 I}}}, i \geq 1, \\
& \frac{\partial \tilde{I}_{010}(\chi, \eta, \phi, \vartheta)}{\partial \vartheta}=\sqrt{\frac{D_{0 I}}{D_{0 V}}}\left[\frac{\partial^{2} \tilde{I}_{010}(\chi, \eta, \phi, \vartheta)}{\partial \chi^{2}}+\frac{\partial^{2} \tilde{I}_{010}(\chi, \eta, \phi, \vartheta)}{\partial \eta^{2}}+\frac{\partial^{2} \tilde{I}_{010}(\chi, \eta, \phi, \vartheta)}{\partial \phi^{2}}\right]- \\
& -\left[1+\varepsilon_{I, V} g_{I, V}(\chi, \eta, \phi, T)\right] \tilde{I}_{000}(\chi, \eta, \phi, \vartheta) \tilde{V}_{000}(\chi, \eta, \phi, \vartheta) \\
& \frac{\partial \tilde{V}_{010}(\chi, \eta, \phi, \vartheta)}{\partial \vartheta}=\sqrt{\frac{D_{0 V}}{D_{0 I}}}\left[\frac{\partial^{2} \tilde{V}_{010}(\chi, \eta, \phi, \vartheta)}{\partial \chi^{2}}+\frac{\partial^{2} \tilde{V}_{010}(\chi, \eta, \phi, \vartheta)}{\partial \eta^{2}}+\frac{\partial^{2} \tilde{V}_{010}(\chi, \eta, \phi, \vartheta)}{\partial \phi^{2}}\right]- \\
& -\left[1+\varepsilon_{I, V} g_{I, V}(\chi, \eta, \phi, T)\right] \tilde{I}_{000}(\chi, \eta, \phi, \vartheta) \tilde{V}_{000}(\chi, \eta, \phi, \vartheta) ; \\
& \frac{\partial \tilde{I}_{020}(\chi, \eta, \phi, \vartheta)}{\partial \vartheta}=\sqrt{\frac{D_{0 I}}{D_{0 V}}}\left[\frac{\partial^{2} \tilde{I}_{020}(\chi, \eta, \phi, \vartheta)}{\partial \chi^{2}}+\frac{\partial^{2} \tilde{I}_{020}(\chi, \eta, \phi, \vartheta)}{\partial \eta^{2}}+\frac{\partial^{2} \tilde{I}_{020}(\chi, \eta, \phi, \vartheta)}{\partial \phi^{2}}\right]- \\
& -\left[1+\varepsilon_{I, V} g_{I, V}(\chi, \eta, \phi, T)\right]\left[\tilde{I}_{010}(\chi, \eta, \phi, \vartheta) \tilde{V}_{000}(\chi, \eta, \phi, \vartheta)+\tilde{I}_{000}(\chi, \eta, \phi, \vartheta) \tilde{V}_{010}(\chi, \eta, \phi, \vartheta)\right] \\
& \frac{\partial \tilde{V}_{020}(\chi, \eta, \phi, \vartheta)}{\partial \vartheta}=\sqrt{\frac{D_{0 I}}{D_{0 V}}}\left[\frac{\partial^{2} \tilde{V}_{020}(\chi, \eta, \phi, \vartheta)}{\partial \chi^{2}}+\frac{\partial^{2} \tilde{V}_{020}(\chi, \eta, \phi, \vartheta)}{\partial \eta^{2}}+\frac{\partial^{2} \tilde{V}_{020}(\chi, \eta, \phi, \vartheta)}{\partial \phi^{2}}\right]- \\
& -\left[1+\varepsilon_{I, V} g_{I, V}(\chi, \eta, \phi, T)\right]\left[\tilde{I}_{010}(\chi, \eta, \phi, \vartheta) \tilde{V}_{000}(\chi, \eta, \phi, \vartheta)+\tilde{I}_{000}(\chi, \eta, \phi, \vartheta) \tilde{V}_{010}(\chi, \eta, \phi, \vartheta)\right] ; \\
& \frac{\partial \tilde{I}_{001}(\chi, \eta, \phi, \vartheta)}{\partial \vartheta}=\sqrt{\frac{D_{0 I}}{D_{0 V}}}\left[\frac{\partial^{2} \tilde{I}_{001}(\chi, \eta, \phi, \vartheta)}{\partial \chi^{2}}+\frac{\partial^{2} \tilde{I}_{001}(\chi, \eta, \phi, \vartheta)}{\partial \eta^{2}}+\frac{\partial^{2} \tilde{I}_{001}(\chi, \eta, \phi, \vartheta)}{\partial \phi^{2}}\right]- \\
& -\left[1+\varepsilon_{I, I} g_{I, I}(\chi, \eta, \phi, T)\right] \tilde{I}_{000}^{2}(\chi, \eta, \phi, \vartheta) \\
& \frac{\partial \tilde{V}_{001}(\chi, \eta, \phi, \vartheta)}{\partial \vartheta}=\sqrt{\frac{D_{0 V}}{D_{0 I}}}\left[\frac{\partial^{2} \tilde{V}_{001}(\chi, \eta, \phi, \vartheta)}{\partial \chi^{2}}+\frac{\partial^{2} \tilde{V}_{001}(\chi, \eta, \phi, \vartheta)}{\partial \eta^{2}}+\frac{\partial^{2} \tilde{V}_{001}(\chi, \eta, \phi, \vartheta)}{\partial \phi^{2}}\right]- \\
& -\left[1+\varepsilon_{I, I} g_{I, I}(\chi, \eta, \phi, T)\right] \tilde{V}_{000}^{2}(\chi, \eta, \phi, \vartheta) ;
\end{aligned}
$$


International Journal on Organic Electronics (IJOE) Vol.6, No.2, April 2017

$$
\begin{aligned}
& \frac{\partial \tilde{I}_{110}(\chi, \eta, \phi, \vartheta)}{\partial \vartheta}=\sqrt{\frac{D_{0 I}}{D_{0 V}}}\left[\frac{\partial^{2} \tilde{I}_{110}(\chi, \eta, \phi, \vartheta)}{\partial \chi^{2}}+\frac{\partial^{2} \tilde{I}_{110}(\chi, \eta, \phi, \vartheta)}{\partial \eta^{2}}+\frac{\partial^{2} \tilde{I}_{110}(\chi, \eta, \phi, \vartheta)}{\partial \phi^{2}}\right]+\sqrt{\frac{D_{0 I}}{D_{0 V}}} \times \\
& \times\left\{\frac{\partial}{\partial \chi}\left[g_{I}(\chi, \eta, \phi, T) \frac{\partial \tilde{I}_{010}(\chi, \eta, \phi, \vartheta)}{\partial \chi}\right]+\frac{\partial}{\partial \eta}\left[g_{I}(\chi, \eta, \phi, T) \frac{\partial \tilde{I}_{010}(\chi, \eta, \phi, \vartheta)}{\partial \eta}\right]+\frac{\partial}{\partial \phi}\left[g_{I}(\chi, \eta, \phi, T) \times\right.\right. \\
& \left.\left.\times \frac{\partial \tilde{I}_{010}(\chi, \eta, \phi, \vartheta)}{\partial \phi}\right]\right\}-\left[\tilde{I}_{100}(\chi, \eta, \phi, \vartheta) \tilde{V}_{000}(\chi, \eta, \phi, \vartheta)+\tilde{I}_{000}(\chi, \eta, \phi, \vartheta) \tilde{V}_{100}(\chi, \eta, \phi, \vartheta)\right] \times \\
& \times\left[1+\varepsilon_{I, I} g_{I, I}(\chi, \eta, \phi, T)\right] \\
& \frac{\partial \tilde{V}_{110}(\chi, \eta, \phi, \vartheta)}{\partial \vartheta}=\sqrt{\frac{D_{0 V}}{D_{0 I}}}\left[\frac{\partial^{2} \tilde{V}_{110}(\chi, \eta, \phi, \vartheta)}{\partial \chi^{2}}+\frac{\partial^{2} \tilde{V}_{110}(\chi, \eta, \phi, \vartheta)}{\partial \eta^{2}}+\frac{\partial^{2} \tilde{V}_{110}(\chi, \eta, \phi, \vartheta)}{\partial \phi^{2}}\right]+\sqrt{\frac{D_{0 V}}{D_{0 I}}} \times \\
& \times\left\{\frac{\partial}{\partial \chi}\left[g_{V}(\chi, \eta, \phi, T) \frac{\partial \tilde{V}_{010}(\chi, \eta, \phi, \vartheta)}{\partial \chi}\right]+\frac{\partial}{\partial \eta}\left[g_{V}(\chi, \eta, \phi, T) \frac{\partial \tilde{V}_{010}(\chi, \eta, \phi, \vartheta)}{\partial \eta}\right]+\frac{\partial}{\partial \phi}\left[g_{I}(\chi, \eta, \phi, T) \times\right.\right. \\
& \left.\left.\times \frac{\partial \tilde{V}_{010}(\chi, \eta, \phi, \vartheta)}{\partial \phi}\right]\right\}-\left[\tilde{V}_{100}(\chi, \eta, \phi, \vartheta) \tilde{I}_{000}(\chi, \eta, \phi, \vartheta)+\tilde{V}_{000}(\chi, \eta, \phi, \vartheta) \tilde{I}_{100}(\chi, \eta, \phi, \vartheta)\right] \times \\
& \times\left\lfloor 1+\varepsilon_{V, V} g_{V, V}(\chi, \eta, \phi, T)\right] ; \\
& \frac{\partial \tilde{I}_{002}(\chi, \eta, \phi, \vartheta)}{\partial \vartheta}=\sqrt{\frac{D_{0 I}}{D_{0 V}}}\left[\frac{\partial^{2} \tilde{I}_{002}(\chi, \eta, \phi, \vartheta)}{\partial \chi^{2}}+\frac{\partial^{2} \tilde{I}_{002}(\chi, \eta, \phi, \vartheta)}{\partial \eta^{2}}+\frac{\partial^{2} \tilde{I}_{002}(\chi, \eta, \phi, \vartheta)}{\partial \phi^{2}}\right]- \\
& -\left[1+\varepsilon_{I, I} g_{I, I}(\chi, \eta, \phi, T)\right] \tilde{I}_{001}(\chi, \eta, \phi, \vartheta) \tilde{I}_{000}(\chi, \eta, \phi, \vartheta) \\
& \frac{\partial \tilde{V}_{002}(\chi, \eta, \phi, \vartheta)}{\partial \vartheta}=\sqrt{\frac{D_{0 V}}{D_{01}}}\left[\frac{\partial^{2} \tilde{V}_{002}(\chi, \eta, \phi, \vartheta)}{\partial \chi^{2}}+\frac{\partial^{2} \tilde{V}_{002}(\chi, \eta, \phi, \vartheta)}{\partial \eta^{2}}+\frac{\partial^{2} \tilde{V}_{002}(\chi, \eta, \phi, \vartheta)}{\partial \phi^{2}}\right]- \\
& -\left[1+\varepsilon_{V, V} g_{V, V}(\chi, \eta, \phi, E)\right] \tilde{V}_{001}(\chi, \eta, \phi, \vartheta) \tilde{V}_{000}(\chi, \eta, \phi, \vartheta) ; \\
& \frac{\partial \tilde{I}_{101}(\chi, \eta, \phi, \vartheta)}{\partial \vartheta}=\sqrt{\frac{D_{0 I}}{D_{0 V}}}\left[\frac{\partial^{2} \tilde{I}_{101}(\chi, \eta, \phi, \vartheta)}{\partial \chi^{2}}+\frac{\partial^{2} \tilde{I}_{101}(\chi, \eta, \phi, \vartheta)}{\partial \eta^{2}}+\frac{\partial^{2} \tilde{I}_{101}(\chi, \eta, \phi, \vartheta)}{\partial \phi^{2}}\right]+ \\
& +\sqrt{\frac{D_{0 I}}{D_{0 V}}}\left\{\frac{\partial}{\partial \chi}\left[g_{I}(\chi, \eta, \phi, T) \frac{\partial \tilde{I}_{001}(\chi, \eta, \phi, \vartheta)}{\partial \chi}\right]+\frac{\partial}{\partial \eta}\left[g_{I}(\chi, \eta, \phi, T) \frac{\partial \tilde{I}_{001}(\chi, \eta, \phi, \vartheta)}{\partial \eta}\right]+\right. \\
& \left.+\frac{\partial}{\partial \phi}\left[g_{I}(\chi, \eta, \phi, T) \frac{\partial \tilde{I}_{001}(\chi, \eta, \phi, \vartheta)}{\partial \phi}\right]\right\}-\left[1+\varepsilon_{I} g_{I}(\chi, \eta, \phi, T)\right] \tilde{I}_{100}(\chi, \eta, \phi, \vartheta) \tilde{V}_{000}(\chi, \eta, \phi, \vartheta) \\
& \frac{\partial \tilde{V}_{101}(\chi, \eta, \phi, \vartheta)}{\partial \vartheta}=\sqrt{\frac{D_{0 V}}{D_{0 I}}}\left[\frac{\partial^{2} \tilde{V}_{101}(\chi, \eta, \phi, \vartheta)}{\partial \chi^{2}}+\frac{\partial^{2} \tilde{V}_{101}(\chi, \eta, \phi, \vartheta)}{\partial \eta^{2}}+\frac{\partial^{2} \tilde{V}_{101}(\chi, \eta, \phi, \vartheta)}{\partial \phi^{2}}\right]+ \\
& +\sqrt{\frac{D_{0 V}}{D_{0 I}}}\left\{\frac{\partial}{\partial \chi}\left[g_{V}(\chi, \eta, \phi, T) \frac{\partial \tilde{V}_{001}(\chi, \eta, \phi, \vartheta)}{\partial \chi}\right]+\frac{\partial}{\partial \eta}\left[g_{V}(\chi, \eta, \phi, T) \frac{\partial \tilde{V}_{001}(\chi, \eta, \phi, \vartheta)}{\partial \eta}\right]+\right. \\
& \left.+\frac{\partial}{\partial \phi}\left[g_{V}(\chi, \eta, \phi, T) \frac{\partial \tilde{V}_{001}(\chi, \eta, \phi, \vartheta)}{\partial \phi}\right]\right\}-\left[1+\varepsilon_{V} g_{V}(\chi, \eta, \phi, T)\right] \tilde{I}_{000}(\chi, \eta, \phi, \vartheta) \tilde{V}_{100}(\chi, \eta, \phi, \vartheta) ; \\
& \frac{\partial \tilde{I}_{011}(\chi, \eta, \phi, \vartheta)}{\partial \vartheta}=\sqrt{\frac{D_{0 I}}{D_{0 V}}}\left[\frac{\partial^{2} \tilde{I}_{011}(\chi, \eta, \phi, \vartheta)}{\partial \chi^{2}}+\frac{\partial^{2} \tilde{I}_{011}(\chi, \eta, \phi, \vartheta)}{\partial \eta^{2}}+\frac{\partial^{2} \tilde{I}_{011}(\chi, \eta, \phi, \vartheta)}{\partial \phi^{2}}\right]-\tilde{I}_{010}(\chi, \eta, \phi, \vartheta) \times
\end{aligned}
$$


International Journal on Organic Electronics (IJOE) Vol.6, No.2, April 2017

$$
\begin{aligned}
& \times\left[1+\varepsilon_{I, I} g_{I, I}(\chi, \eta, \phi, T)\right] \tilde{I}_{000}(\chi, \eta, \phi, \vartheta)-\left[1+\varepsilon_{I, V} g_{I, V}(\chi, \eta, \phi, T)\right] \tilde{I}_{001}(\chi, \eta, \phi, \vartheta) \tilde{V}_{000}(\chi, \eta, \phi, \vartheta) \\
& \frac{\partial \tilde{V}_{011}(\chi, \eta, \phi, \vartheta)}{\partial \vartheta}=\sqrt{\frac{D_{0 V}}{D_{0 I}}}\left[\frac{\partial^{2} \tilde{V}_{011}(\chi, \eta, \phi, \vartheta)}{\partial \chi \chi^{2}}+\frac{\partial^{2} \tilde{V}_{011}(\chi, \eta, \phi, \vartheta)}{\partial \eta^{2}}+\frac{\partial^{2} \tilde{V}_{011}(\chi, \eta, \phi, \vartheta)}{\partial \phi^{2}}\right]-\tilde{V}_{010}(\chi, \eta, \phi, \vartheta) \times \\
& \times\left[1+\varepsilon_{V, V} g_{V, V}(\chi, \eta, \phi, T)\right] \tilde{V}_{000}(\chi, \eta, \phi, \vartheta)-\left[1+\varepsilon_{I, V} g_{I, V}(\chi, \eta, \phi, t)\right] \tilde{I}_{000}(\chi, \eta, \phi, \vartheta) \tilde{V}_{001}(\chi, \eta, \phi, \vartheta) ; \\
& \left.\frac{\partial \tilde{\rho}_{i j k}(\chi, \eta, \phi, \vartheta)}{\partial \chi}\right|_{x=0}=0,\left.\frac{\partial \tilde{\rho}_{i j k}(\chi, \eta, \phi, \vartheta)}{\partial \chi}\right|_{x=1}=0,\left.\frac{\partial \tilde{\rho}_{i j k}(\chi, \eta, \phi, \vartheta)}{\partial \eta}\right|_{\eta=0}=0,\left.\frac{\partial \tilde{\rho}_{i j k}(\chi, \eta, \phi, \vartheta)}{\partial \eta}\right|_{\eta=1}=0, \\
& \left.\frac{\partial \tilde{\rho}_{i j k}(\chi, \eta, \phi, \vartheta)}{\partial \phi}\right|_{\phi=0}=0,\left.\frac{\partial \tilde{\rho}_{i j k}(\chi, \eta, \phi, \vartheta)}{\partial \phi}\right|_{\phi=1}=0(i \geq 0, j \geq 0, k \geq 0) ; \\
& \tilde{\rho}_{000}(\chi, \eta, \phi, 0)=f_{\rho}(\chi, \eta, \phi) / \rho^{*}, \tilde{\rho}_{i j k}(\chi, \eta, \phi, 0)=0(i \geq 1, j \geq 1, k \geq 1) .
\end{aligned}
$$

Solutions of the above equations could be written as

$$
\tilde{\rho}_{000}(\chi, \eta, \phi, \vartheta)=\frac{1}{L}+\frac{2}{L} \sum_{n=1}^{\infty} F_{n \rho} c(\chi) c(\eta) c(\phi) e_{n \rho}(\vartheta),
$$

where $F_{n \rho}=\frac{1}{\rho^{*}} \int_{0}^{1} \cos (\pi n u) \int_{0}^{1} \cos (\pi n v) \int_{0}^{1} \cos (\pi n w) f_{n \rho}(u, v, w) d w d v d u, \quad e_{n I}(\vartheta)=\exp \left(-\pi^{2} n^{2} \vartheta \sqrt{D_{0 V} / D_{0 I}}\right)$, $c_{n}(\chi)=\cos (\pi n \chi), e_{n V}(\vartheta)=\exp \left(-\pi^{2} n^{2} \vartheta \sqrt{D_{0 I} / D_{0 V}}\right)$;

$\tilde{I}_{i 00}(\chi, \eta, \phi, \vartheta)=-2 \pi \sqrt{\frac{D_{0 I}}{D_{0 V}}} \sum_{n=1}^{\infty} n c_{n}(\chi) c(\eta) c(\phi) e_{n I}(\vartheta) \int_{0}^{\vartheta} e_{n I}(-\tau) \int_{0}^{1} s_{n}(u) \int_{0}^{1} c_{n}(v) \int_{0}^{1} \frac{\partial \tilde{I}_{i-100}(u, v, w, \tau)}{\partial u} \times$ $\times c_{n}(w) g_{I}(u, v, w, T) d w d v d u d \tau-2 \pi \sqrt{\frac{D_{0 I}}{D_{0 V}}} \sum_{n=1}^{\infty} n c_{n}(\chi) c(\eta) c(\phi) e_{n I}(\vartheta) \int_{0}^{\vartheta} e_{n I}(-\tau) \int_{0}^{1} c_{n}(u) \int_{0}^{1} s_{n}(v) \times$ $\times \int_{0}^{1} c_{n}(w) g_{I}(u, v, w, T) \frac{\partial \tilde{I}_{i-100}(u, v, w, \tau)}{\partial v} d w d v d u d \tau-2 \pi \sqrt{\frac{D_{0 I}}{D_{0 V}}} \sum_{n=1}^{\infty} n c_{n}(\chi) c(\eta) c(\phi) e_{n I}(\vartheta) \int_{0}^{\vartheta} e_{n I}(-\tau) \times$ $\times \int_{0}^{1} c_{n}(u) \int_{0}^{1} c_{n}(v) \int_{0}^{1} s_{n}(w) g_{I}(u, v, w, T) \frac{\partial \tilde{I}_{i-100}(u, v, w, \tau)}{\partial w} d w d v d u d \tau, i \geq 1$, $\tilde{V}_{i 00}(\chi, \eta, \phi, \vartheta)=-2 \pi \sqrt{\frac{D_{0 V}}{D_{0 I}}} \sum_{n=1}^{\infty} n c_{n}(\chi) c(\eta) c(\phi) e_{n V}(\vartheta) \int_{0}^{\vartheta} e_{n I}(-\tau) \int_{0}^{1} s_{n}(u) \int_{0}^{1} c_{n}(v) \int_{0}^{1} g_{V}(u, v, w, T) \times$ $\times c_{n}(w) \frac{\partial \tilde{V}_{i-100}(u, \tau)}{\partial u} d w d v d u d \tau-\sqrt{\frac{D_{0 V}}{D_{0 I}}} \sum_{n=1}^{\infty} n c_{n}(\chi) c(\eta) c(\phi) e_{n V}(\vartheta) \int_{0}^{\vartheta} e_{n I}(-\tau) \int_{0}^{1} c_{n}(u) \int_{0}^{1} s_{n}(v) \times$ $\times 2 \pi \int_{0}^{1} c_{n}(w) g_{V}(u, v, w, T) \frac{\partial \tilde{V}_{i-100}(u, \tau)}{\partial v} d w d v d u d \tau-2 \pi \sqrt{\frac{D_{0 V}}{D_{0 I}}} \sum_{n=1}^{\infty} n c_{n}(\chi) c(\eta) c(\phi) e_{n V}(\vartheta) \times$ $\times \int_{0}^{\vartheta} e_{n I}(-\tau) \int_{0}^{1} c_{n}(u) \int_{0}^{1} c_{n}(v) \int_{0}^{1} s_{n}(w) g_{V}(u, v, w, T) \frac{\partial \tilde{V}_{i-100}(u, \tau)}{\partial w} d w d v d u d \tau, i \geq 1$,

where $s_{n}(\chi)=\sin (\pi n \chi)$;

$\tilde{\rho}_{010}(\chi, \eta, \phi, \vartheta)=-2 \sum_{n=1}^{\infty} c_{n}(\chi) c_{n}(\eta) c_{n}(\phi) e_{n \rho}(\vartheta) \int_{0}^{\vartheta} e_{n \rho}(-\tau) \int_{0}^{1} c_{n}(u) \int_{0}^{1} c_{n}(v) \int_{0}^{1} c_{n}(w) \times$ $\times\left[1+\varepsilon_{I, V} g_{I, V}(u, v, w, T)\right] \tilde{I}_{000}(u, v, w, \tau) \tilde{V}_{000}(u, v, w, \tau) d w d v d u d \tau ;$

$\tilde{\rho}_{020}(\chi, \eta, \phi, \vartheta)=-2 \sqrt{\frac{D_{0 I}}{D_{0 V}}} \sum_{n=1}^{\infty} c_{n}(\chi) c_{n}(\eta) c_{n}(\phi) e_{n \rho}(\vartheta) \int_{0}^{\vartheta} e_{n \rho}(-\tau) \int_{0}^{1} c_{n}(u) \int_{0}^{1} c_{n}(v) \int_{0}^{1} c_{n}(w)\left[1+\varepsilon_{I, V} \times\right.$ $\left.\times g_{I, V}(u, v, w, T)\right]\left[\tilde{I}_{010}(u, v, w, \tau) \tilde{V}_{000}(u, v, w, \tau)+\tilde{I}_{000}(u, v, w, \tau) \tilde{V}_{010}(u, v, w, \tau)\right] d w d v d u d \tau ;$ 
International Journal on Organic Electronics (IJOE) Vol.6, No.2, April 2017

$$
\begin{aligned}
& \widetilde{\rho}_{001}(\chi, \eta, \phi, \vartheta)=-2 \sum_{n=1}^{\infty} c_{n}(\chi) c_{n}(\eta) c_{n}(\phi) e_{n \rho}(\vartheta) \int_{0}^{\vartheta} e_{n \rho}(-\tau) \int_{0}^{1} c_{n}(u) \int_{0}^{1} c_{n}(v) \int_{0}^{1} c_{n}(w) \times \\
& \times\left[1+\varepsilon_{\rho, \rho} g_{\rho, \rho}(u, v, w, T)\right] \tilde{\rho}_{000}^{2}(u, v, w, \tau) d w d v d u d \tau ; \\
& \tilde{\rho}_{002}(\chi, \eta, \phi, \vartheta)=-2 \sum_{n=1}^{\infty} c_{n}(\chi) c_{n}(\eta) c_{n}(\phi) e_{n \rho}(\vartheta) \int_{0}^{\vartheta} e_{n \rho}(-\tau) \int_{0}^{1} c_{n}(u) \int_{0}^{1} c_{n}(v) \int_{0}^{1} c_{n}(w) \times \\
& \times\left[1+\varepsilon_{\rho, \rho} g_{\rho, \rho}(u, v, w, T)\right] \tilde{\rho}_{001}(u, v, w, \tau) \tilde{\rho}_{000}(u, v, w, \tau) d w d v d u d \tau ; \\
& \tilde{I}_{110}(\chi, \eta, \phi, \vartheta)=-2 \pi \sqrt{\frac{D_{0 I}}{D_{0 V}}} \sum_{n=1}^{\infty} n c_{n}(\chi) c_{n}(\eta) c_{n}(\phi) e_{n I}(\vartheta) \int_{0}^{\vartheta} e_{n I}(-\tau) \int_{0}^{1} s_{n}(u) \int_{0}^{1} c_{n}(v) \int_{0}^{1} c_{n}(u) \times \\
& \times g_{I}(u, v, w, T) \frac{\partial \tilde{I}_{i-100}(u, v, w, \tau)}{\partial u} d w d v d u d \tau-2 \pi \sqrt{\frac{D_{0 I}}{D_{0 V}}} \sum_{n=1}^{\infty} n c_{n}(\chi) c_{n}(\eta) c_{n}(\phi) e_{n I}(\vartheta) \times \\
& \times \int_{0}^{\vartheta} e_{n I}(-\tau) \int_{0}^{1} c_{n}(u) \int_{0}^{1} s_{n}(v) \int_{0}^{1} c_{n}(u) g_{I}(u, v, w, T) \frac{\partial \tilde{I}_{i-100}(u, v, w, \tau)}{\partial v} d w d v d u d \tau-2 \pi \sqrt{\frac{D_{0 I}}{D_{0 V}}} \times \\
& \times \sum_{n=1}^{\infty} n e_{n I}(\vartheta) \int_{0}^{\vartheta} e_{n I}(-\tau) \int_{0}^{1} c_{n}(u) \int_{0}^{1} c_{n}(v) \int_{0}^{1} s_{n}(u) g_{I}(u, v, w, T) \frac{\partial \tilde{I}_{i-100}(u, v, w, \tau)}{\partial w} d w d v d u d \tau \times \\
& \times c_{n}(\chi) c_{n}(\eta) c_{n}(\phi)-2 \sum_{n=1}^{\infty} c_{n}(\chi) e_{n I}(\vartheta) c_{n}(\eta) c_{n}(\phi) \int_{0}^{\vartheta} e_{n I}(-\tau) \int_{0}^{1} c_{n}(u) \int_{0}^{1} c_{n}(v) \int_{0}^{1} c_{n}(v)\left[1+\varepsilon_{I, V} \times\right. \\
& \left.\times g_{I, V}(u, v, w, T)\right]\left[\tilde{I}_{100}(u, v, w, \tau) \tilde{V}_{000}(u, v, w, \tau)+\tilde{I}_{000}(u, v, w, \tau) \tilde{V}_{100}(u, v, w, \tau)\right] d w d v d u d \tau \\
& \tilde{V}_{110}(\chi, \eta, \phi, \vartheta)=-2 \pi \sqrt{\frac{D_{0 V}}{D_{0 I}}} \sum_{n=1}^{\infty} n c_{n}(\chi) c_{n}(\eta) c_{n}(\phi) e_{n V}(\vartheta) \int_{0}^{\vartheta} e_{n V}(-\tau) \int_{0}^{1} s_{n}(u) \int_{0}^{1} c_{n}(v) \int_{0}^{1} c_{n}(u) \times \\
& \times g_{V}(u, v, w, T) \frac{\partial \tilde{V}_{i-100}(u, v, w, \tau)}{\partial u} d w d v d u d \tau-2 \pi \sqrt{\frac{D_{0 V}}{D_{0 I}}} \sum_{n=1}^{\infty} n c_{n}(\chi) c_{n}(\eta) c_{n}(\phi) e_{n V}(\vartheta) \times \\
& \times \int_{0}^{\vartheta} e_{n V}(-\tau) \int_{0}^{1} c_{n}(u) \int_{0}^{1} s_{n}(v) \int_{0}^{1} c_{n}(u) g_{V}(u, v, w, T) \frac{\partial \tilde{V}_{i-100}(u, v, w, \tau)}{\partial v} d w d v d u d \tau-2 \pi \sqrt{\frac{D_{0 V}}{D_{0 I}}} \times \\
& \times \sum_{n=1}^{\infty} n e_{n V}(\vartheta) \int_{0}^{\vartheta} e_{n V}(-\tau) \int_{0}^{1} c_{n}(u) \int_{0}^{1} c_{n}(v) \int_{0}^{1} s_{n}(u) g_{V}(u, v, w, T) \frac{\partial \tilde{V}_{i-100}(u, v, w, \tau)}{\partial w} d w d v d u d \tau \times \\
& \times c_{n}(\chi) c_{n}(\eta) c_{n}(\phi)-2 \sum_{n=1}^{\infty} c_{n}(\chi) e_{n I}(\vartheta) c_{n}(\eta) c_{n}(\phi) \int_{0}^{\vartheta} e_{n V}(-\tau) \int_{0}^{1} c_{n}(u) \int_{0}^{1} c_{n}(v) \int_{0}^{1}\left[1+\varepsilon_{I, V} g_{I, V}(u, v, w, T)\right] \times \\
& \times c_{n}(w)\left[\tilde{I}_{100}(u, v, w, \tau) \tilde{V}_{000}(u, v, w, \tau)+\tilde{I}_{000}(u, v, w, \tau) \tilde{V}_{100}(u, v, w, \tau)\right] d w d v d u d \tau ; \\
& \tilde{I}_{101}(\chi, \eta, \phi, \vartheta)=-2 \pi \sqrt{\frac{D_{0 I}}{D_{0 V}}} \sum_{n=1}^{\infty} n c_{n}(\chi) c_{n}(\eta) c_{n}(\phi) e_{n I}(\vartheta) \int_{0}^{\vartheta} e_{n I}(-\tau) \int_{0}^{1} s_{n}(u) \int_{0}^{1} c_{n}(v) \int_{0}^{1} g_{I}(u, v, w, T) \times \\
& \times c_{n}(w) \frac{\partial \tilde{I}_{001}(u, v, w, \tau)}{\partial u} d w d v d u d \tau-2 \pi \sqrt{\frac{D_{0 I}}{D_{0 V}}} \sum_{n=1}^{\infty} n c_{n}(\chi) c_{n}(\eta) c_{n}(\phi) e_{n I}(\vartheta) \int_{0}^{\vartheta} e_{n I}(-\tau) \int_{0}^{1} c_{n}(u) \times \\
& \times \int_{0}^{1} s_{n}(v) \int_{0}^{1} c_{n}(w) g_{I}(u, v, w, T) \frac{\partial \tilde{I}_{001}(u, v, w, \tau)}{\partial v} d w d v d u d \tau-2 \pi \sqrt{\frac{D_{0 I}}{D_{0 V}}} \sum_{n=1}^{\infty} n e_{n I}(\vartheta) c_{n}(\chi) c_{n}(\eta) c_{n}(\phi) \times \\
& \times \int_{0}^{\vartheta} e_{n I}(-\tau) \int_{0}^{1} c_{n}(u) \int_{0}^{1} c_{n}(v) \int_{0}^{1} s_{n}(w) g_{I}(u, v, w, T) \frac{\partial \tilde{I}_{001}(u, v, w, \tau)}{\partial w} d w d v d u d \tau-2 \sum_{n=1}^{\infty} c_{n}(\chi) c_{n}(\eta) c_{n}(\phi) \times \\
& \times e_{n I}(\vartheta) \int_{0}^{\vartheta} e_{n I}(-\tau) \int_{0}^{1} c_{n}(u) \int_{0}^{1} c_{n}(v) \int_{0}^{1} c_{n}(w)\left[1+\varepsilon_{I, V} g_{I, V}(u, v, w, T)\right] \tilde{I}_{100}(u, v, w, \tau) \tilde{V}_{000}(u, v, w, \tau) d w d v d u d \tau
\end{aligned}
$$


$\tilde{V}_{101}(\chi, \eta, \phi, \vartheta)=-2 \pi \sqrt{\frac{D_{0 V}}{D_{0 I}}} \sum_{n=1}^{\infty} n c_{n}(\chi) c_{n}(\eta) c_{n}(\phi) e_{n V}(\vartheta) \int_{0}^{\vartheta} e_{n V}(-\tau) \int_{0}^{1} s_{n}(u) \int_{0}^{1} c_{n}(v) \int_{0}^{1} g_{V}(u, v, w, T) \times$ $\times c_{n}(w) \frac{\partial \tilde{V}_{001}(u, v, w, \tau)}{\partial u} d w d v d u d \tau-2 \pi \sqrt{\frac{D_{0 V}}{D_{0 I}}} \sum_{n=1}^{\infty} n c_{n}(\chi) c_{n}(\eta) c_{n}(\phi) e_{n I}(\vartheta) \int_{0}^{\vartheta} e_{n V}(-\tau) \int_{0}^{1} c_{n}(u) \times$ $\times \int_{0}^{1} s_{n}(v) \int_{0}^{1} c_{n}(w) g_{V}(u, v, w, T) \frac{\partial \tilde{V}_{001}(u, v, w, \tau)}{\partial v} d w d v d u d \tau-2 \pi \sqrt{\frac{D_{0 V}}{D_{0 I}}} \sum_{n=1}^{\infty} n e_{n V}(\vartheta) c_{n}(\chi) c_{n}(\eta) c_{n}(\phi) \times$ $\times \int_{0}^{\vartheta} e_{n V}(-\tau) \int_{0}^{1} c_{n}(u) \int_{0}^{1} c_{n}(v) \int_{0}^{1} s_{n}(w) g_{V}(u, v, w, T) \frac{\partial \tilde{V}_{001}(u, v, w, \tau)}{\partial w} d w d v d u d \tau-2 \sum_{n=1}^{\infty} c_{n}(\chi) c_{n}(\eta) c_{n}(\phi) \times$ $\times e_{n V}(\vartheta) \int_{0}^{\vartheta} e_{n V}(-\tau) \int_{0}^{1} c_{n}(u) \int_{0}^{1} c_{n}(v) \int_{0}^{1} c_{n}(w)\left[1+\varepsilon_{I, V} g_{I, V}(u, v, w, T)\right] \tilde{I}_{100}(u, v, w, \tau) \tilde{V}_{000}(u, v, w, \tau) d w d v d u d \tau ;$ $\tilde{I}_{011}(\chi, \eta, \phi, \vartheta)=-2 \sum_{n=1}^{\infty} c_{n}(\chi) c_{n}(\eta) c_{n}(\phi) e_{n I}(\vartheta) \int_{0}^{\vartheta} e_{n I}(-\tau) \int_{0}^{1} c_{n}(u) \int_{0}^{1} c_{n}(v) \int_{0}^{1} c_{n}(w)\left\{\tilde{I}_{000}(u, v, w, \tau) \times\right.$ $\left.\times\left[1+\varepsilon_{I, I} g_{I, I}(u, v, w, T)\right] \tilde{I}_{010}(u, v, w, \tau)+\left[1+\varepsilon_{I, V} g_{I, V}(u, v, w, T)\right] \tilde{I}_{001}(u, v, w, \tau) \tilde{V}_{000}(u, v, w, \tau)\right\} d w d v d u d \tau$ $\tilde{V}_{011}(\chi, \eta, \phi, \vartheta)=-2 \sum_{n=1}^{\infty} c_{n}(\chi) c_{n}(\eta) c_{n}(\phi) e_{n V}(\vartheta) \int_{0}^{\vartheta} e_{n V}(-\tau) \int_{0}^{1} c_{n}(u) \int_{0}^{1} c_{n}(v) \int_{0}^{1} c_{n}(w)\left\{\tilde{I}_{000}(u, v, w, \tau) \times\right.$ $\left.\times\left[1+\varepsilon_{V, V} g_{V, V}(u, v, w, T)\right] \tilde{I}_{010}(u, v, w, \tau)+\left[1+\varepsilon_{I, V} g_{I, V}(u, v, w, T)\right] \tilde{I}_{001}(u, v, w, \tau) \tilde{V}_{000}(u, v, w, \tau)\right\} d w d v d u d \tau$.

Equations for the functions $\Phi_{\rho_{i}}(x, y, z, t)$, boundary and initial conditions for them could be written as

$$
\begin{aligned}
& \frac{\partial \Phi_{I 0}(x, y, z, t)}{\partial t}=D_{0 \Phi I}\left[\frac{\partial^{2} \Phi_{I 0}(x, y, z, t)}{\partial x^{2}}+\frac{\partial^{2} \Phi_{I 0}(x, y, z, t)}{\partial y^{2}}+\frac{\partial^{2} \Phi_{I 0}(x, y, z, t)}{\partial z^{2}}\right]+ \\
& +k_{I, I}(x, y, z, T) I^{2}(x, y, z, t)-k_{I}(x, y, z, T) I(x, y, z, t) \\
& \frac{\partial \Phi_{V 0}(x, y, z, t)}{\partial t}=D_{0 \Phi V}\left[\frac{\partial^{2} \Phi_{V 0}(x, y, z, t)}{\partial x^{2}}+\frac{\partial^{2} \Phi_{V 0}(x, y, z, t)}{\partial y^{2}}+\frac{\partial^{2} \Phi_{V 0}(x, y, z, t)}{\partial z^{2}}\right]+ \\
& +k_{V, V}(x, y, z, T) V^{2}(x, y, z, t)-k_{V}(x, y, z, T) V(x, y, z, t) ; \\
& \frac{\partial \Phi_{I i}(x, y, z, t)}{\partial t}=D_{0 \Phi I}\left[\frac{\partial^{2} \Phi_{I i}(x, y, z, t)}{\partial x^{2}}+\frac{\partial^{2} \Phi_{I i}(x, y, z, t)}{\partial y^{2}}+\frac{\partial^{2} \Phi_{I i}(x, y, z, t)}{\partial z^{2}}\right]+ \\
& +D_{0 \Phi I}\left\{\frac{\partial}{\partial x}\left[g_{\Phi I}(x, y, z, T) \frac{\partial \Phi_{I i-1}(x, y, z, t)}{\partial x}\right]+\frac{\partial}{\partial y}\left[g_{\Phi I}(x, y, z, T) \frac{\partial \Phi_{I i-1}(x, y, z, t)}{\partial y}\right]+\right. \\
& \left.+\frac{\partial}{\partial z}\left[g_{\Phi I}(x, y, z, T) \frac{\partial \Phi_{I i-1}(x, y, z, t)}{\partial z}\right]\right\}, i \geq 1, \\
& \frac{\partial \Phi_{V i}(x, y, z, t)}{\partial t}=D_{0 \Phi V}\left[\frac{\partial^{2} \Phi_{V i}(x, y, z, t)}{\partial x^{2}}+\frac{\partial^{2} \Phi_{V i}(x, y, z, t)}{\partial y^{2}}+\frac{\partial^{2} \Phi_{V i}(x, y, z, t)}{\partial z^{2}}\right]+ \\
& +D_{0 \Phi V}\left\{\frac{\partial}{\partial x}\left[g_{\Phi V}(x, y, z, T) \frac{\partial \Phi_{V i-1}(x, y, z, t)}{\partial x}\right]+\frac{\partial}{\partial y}\left[g_{\Phi V}(x, y, z, T) \frac{\partial \Phi_{V i-1}(x, y, z, t)}{\partial y}\right]+\right. \\
& \left.+\frac{\partial}{\partial z}\left[g_{\Phi V}(x, y, z, T) \frac{\partial \Phi_{V i-1}(x, y, z, t)}{\partial z}\right]\right\}, i \geq 1 ; \\
& \left.\frac{\partial \Phi_{\rho i}(x, y, z, t)}{\partial x}\right|_{x=0}=0,\left.\frac{\partial \Phi_{\rho i}(x, y, z, t)}{\partial x}\right|_{x=L_{x}}=0,\left.\frac{\partial \Phi_{\rho i}(x, y, z, t)}{\partial y}\right|_{y=0}=0,\left.\frac{\partial \Phi_{\rho i}(x, y, z, t)}{\partial y}\right|_{y=L_{y}}=0 \text {, }
\end{aligned}
$$


International Journal on Organic Electronics (IJOE) Vol.6, No.2, April 2017

$$
\left.\frac{\partial \Phi_{\rho i}(x, y, z, t)}{\partial z}\right|_{z=0}=0,\left.\frac{\partial \Phi_{\rho i}(x, y, z, t)}{\partial z}\right|_{z=L_{z}}=0, i \geq 0 ; \Phi_{\rho 0}(x, y, z, 0)=f_{\Phi \rho}(x, y, z), \Phi_{\rho i}(x, y, z, 0)=0, i \geq 1 .
$$

Solutions of the above equations could be written as

$$
\begin{aligned}
& \Phi_{\rho 0}(x, y, z, t)=\frac{1}{L_{x} L_{y} L_{z}}+\frac{2}{L_{x} L_{y} L_{z}} \sum_{n=1}^{\infty} F_{n \Phi_{\rho}} c_{n}(x) c_{n}(y) c_{n}(z) e_{n \Phi_{\rho}}(t)+\frac{2}{L} \sum_{n=1}^{\infty} n c_{n}(x) c_{n}(y) c_{n}(z) \times \\
& \times e_{\Phi_{\rho^{n}}}(t) \int_{0}^{t} e_{\Phi_{\rho^{n}}}(-\tau) \int_{0}^{L_{x}} c_{n}(u) \int_{0}^{L_{y}} c_{n}(v) \int_{0}^{L_{z}} c_{n}(w)\left[k_{I, I}(u, v, w, T) I^{2}(u, v, w, \tau)-\right.
\end{aligned}
$$

$\left.-k_{I}(u, v, w, T) I(u, v, w, \tau)\right] d w d v d u d \tau$,

where $\quad F_{n \Phi_{\rho}}=\int_{0}^{L_{x}} c_{n}(u) \int_{0}^{L_{y}} c_{n}(v) \int_{0}^{L_{z}} c_{n}(w) f_{\Phi_{\rho}}(u, v, w) d w d v d u, \quad e_{n \Phi_{\rho}}(t)=\exp \left[-\pi^{2} n^{2} D_{0 \Phi_{\rho}} t\left(\frac{1}{L_{x}^{2}}+\frac{1}{L_{y}^{2}}+\frac{1}{L_{z}^{2}}\right)\right]$, $c_{n}(x)=\cos \left(\pi n x / L_{x}\right)$;

$$
\begin{gathered}
\Phi_{\rho i}(x, y, z, t)=-\frac{2 \pi}{L_{x}^{2} L_{y} L_{z}} \sum_{n=1}^{\infty} n c_{n}(x) c_{n}(y) c_{n}(z) e_{\Phi_{\rho^{n}}}(t) \int_{0}^{t} e_{\Phi_{\rho^{n}}}(-\tau) \int_{0}^{L_{x}} s_{n}(u) \int_{0}^{L_{y}} c_{n}(v) \int_{0}^{L_{z}} g_{\Phi_{\rho}}(u, v, w, T) \times \\
\times c_{n}(w) \frac{\partial \Phi_{I_{\rho} i-1}(u, v, w, \tau)}{\partial u} d w d v d u d \tau-\frac{2 \pi}{L_{x} L_{y}^{2} L_{z}} \sum_{n=1}^{\infty} n c_{n}(x) c_{n}(y) c_{n}(z) e_{\Phi_{\rho^{n}}}(t) \int_{0}^{t} e_{\Phi_{\rho^{n}}}(-\tau) \times \\
\times \int_{0}^{t} e_{\Phi_{\rho^{n}}}(-\tau) \int_{0}^{L_{x}} c_{n}(u) \int_{0}^{L_{y}} s_{n}(v) \int_{0}^{L_{z}} c_{n}(w) g_{\Phi_{\rho}}(u, v, w, T) \frac{\partial \Phi_{I_{\rho} i-1}(u, v, w, \tau)}{\partial v} d w d v d u d \tau-\frac{2 \pi}{L_{x} L_{y} L_{z}^{2}} \sum_{n=1}^{\infty} n \times \\
\times e_{\Phi_{\rho^{n}}}(t) \int_{0}^{t} e_{\Phi_{\rho^{n}}}(-\tau) \int_{0}^{L_{x}} c_{n}(u) \int_{0}^{L_{y}} c_{n}(v) \int_{0}^{L_{z}} s_{n}(w) \frac{\partial \Phi_{I_{\rho} i-1}(u, v, w, \tau)}{\partial w} g_{\Phi_{\rho}}(u, v, w, T) d w d v d u d \tau \times \\
\times c_{n}(x) c_{n}(y) c_{n}(z), i \geq 1,
\end{gathered}
$$

where $s_{n}(x)=\sin \left(\pi n x / L_{x}\right)$.

Equations for the functions $C_{i j}(x, y, z, t)$, boundary and initial conditions for them could be written as

$$
\begin{aligned}
& \frac{\partial C_{00}(x, y, z, t)}{\partial t}=D_{0 L} \frac{\partial^{2} C_{00}(x, y, z, t)}{\partial x^{2}}+D_{0 L} \frac{\partial^{2} C_{00}(x, y, z, t)}{\partial y^{2}}+D_{0 L} \frac{\partial^{2} C_{00}(x, y, z, t)}{\partial z^{2}} ; \\
& \frac{\partial C_{i 0}(x, y, z, t)}{\partial t}=D_{0 L}\left[\frac{\partial^{2} C_{i 0}(x, y, z, t)}{\partial x^{2}}+\frac{\partial^{2} C_{i 0}(x, y, z, t)}{\partial y^{2}}+\frac{\partial^{2} C_{i 0}(x, y, z, t)}{\partial z^{2}}\right]+ \\
& +D_{0 L} \frac{\partial}{\partial x}\left[g_{L}(x, y, z, T) \frac{\partial C_{i-10}(x, y, z, t)}{\partial x}\right]+D_{0 L} \frac{\partial}{\partial y}\left[g_{L}(x, y, z, T) \frac{\partial C_{i-10}(x, y, z, t)}{\partial y}\right]+ \\
& +D_{0 L} \frac{\partial}{\partial z}\left[g_{L}(x, y, z, T) \frac{\partial C_{i-10}(x, y, z, t)}{\partial z}\right], i \geq 1 \\
& \frac{\partial C_{01}(x, y, z, t)}{\partial t}=D_{0 L} \frac{\partial^{2} C_{01}(x, y, z, t)}{\partial x^{2}}+D_{0 L} \frac{\partial^{2} C_{01}(x, y, z, t)}{\partial y^{2}}+D_{0 L} \frac{\partial^{2} C_{01}(x, y, z, t)}{\partial z^{2}}+ \\
& +D_{0 L} \frac{\partial}{\partial x}\left[\frac{C_{00}^{\gamma}(x, y, z, t)}{P^{\gamma}(x, y, z, T)} \frac{\partial C_{00}(x, y, z, t)}{\partial x}\right]+D_{0 L} \frac{\partial}{\partial y}\left[\frac{C_{00}^{\gamma}(x, y, z, t)}{P^{\gamma}(x, y, z, T)} \frac{\partial C_{00}(x, y, z, t)}{\partial y}\right]+ \\
& +D_{0 L} \frac{\partial}{\partial z}\left[\frac{C_{00}^{\gamma}(x, y, z, t)}{P^{\gamma}(x, y, z, T)} \frac{\partial C_{00}(x, y, z, t)}{\partial z}\right] ; \\
& \frac{\partial C_{02}(x, y, z, t)}{\partial t}=D_{0 L} \frac{\partial^{2} C_{02}(x, y, z, t)}{\partial x^{2}}+D_{0 L} \frac{\partial^{2} C_{02}(x, y, z, t)}{\partial y^{2}}+D_{0 L} \frac{\partial^{2} C_{02}(x, y, z, t)}{\partial z^{2}}+
\end{aligned}
$$


International Journal on Organic Electronics (IJOE) Vol.6, No.2, April 2017

$$
\begin{gathered}
+D_{0 L}\left\{\frac{\partial}{\partial x}\left[C_{01}(x, y, z, t) \frac{C_{00}^{\gamma-1}(x, y, z, t)}{P^{\gamma}(x, y, z, T)} \frac{\partial C_{00}(x, y, z, t)}{\partial x}\right]+\frac{\partial}{\partial y}\left[C_{01}(x, y, z, t) \frac{C_{00}^{\gamma-1}(x, y, z, t)}{P^{\gamma}(x, y, z, T)} \times\right.\right. \\
\left.\left.\times \frac{\partial C_{00}(x, y, z, t)}{\partial y}\right]+\frac{\partial}{\partial z}\left[C_{01}(x, y, z, t) \frac{C_{00}^{\gamma-1}(x, y, z, t)}{P^{\gamma}(x, y, z, T)} \frac{\partial C_{00}(x, y, z, t)}{\partial z}\right]\right\}+ \\
\left.\left.\times \frac{\partial C_{00}(x, y, z, t)}{\partial y}\right]+\frac{\partial}{\partial z}\left[C_{01}(x, y, z, t) \frac{C_{00}^{\gamma-1}(x, y, z, t)}{P^{\gamma}(x, y, z, T)} \frac{\partial C_{00}(x, y, z, t)}{\partial z}\right]\right\}+D_{0 L}\left\{\frac { \partial } { \partial x } \left[\frac{C_{00}^{\gamma}(x, y, z, t)}{P^{\gamma}(x, y, z, T)} \times\right.\right. \\
\left.\left.\times \frac{\partial C_{01}(x, y, z, t)}{\partial x}\right]+\frac{\partial}{\partial y}\left[\frac{C_{00}^{\gamma}(x, y, z, t)}{P^{\gamma}(x, y, z, T)} \frac{\partial C_{01}(x, y, z, t)}{\partial y}\right]+\frac{\partial}{\partial z}\left[\frac{C_{00}^{\gamma}(x, y, z, t)}{P^{\gamma}(x, y, z, T)} \frac{\partial C_{01}(x, y, z, t)}{\partial z}\right]\right\} ; \\
\frac{\partial C_{11}(x, y, z, t)}{\partial t}=D_{0 L} \frac{\partial^{2} C_{11}(x, y, z, t)}{\partial x^{2}}+D_{0 L} \frac{\partial^{2} C_{11}(x, y, z, t)}{\partial y^{2}}+D_{0 L} \frac{\partial^{2} C_{11}(x, y, z, t)}{\partial z^{2}}+ \\
+\left\{\frac{\partial}{\partial x}\left[C_{10}(x, y, z, t) \frac{C_{00}^{\gamma-1}(x, y, z, t)}{P^{\gamma}(x, y, z, T)} \frac{\partial C_{00}(x, y, z, t)}{\partial x}\right]+\frac{\partial}{\partial y}\left[C_{10}(x, y, z, t) \frac{C_{00}^{\gamma-1}(x, y, z, t)}{P^{\gamma}(x, y, z, T)} \times\right.\right. \\
\left.\left.\times \frac{\partial C_{00}(x, y, z, t)}{\partial y}\right]+\frac{\partial}{\partial z}\left[C_{10}(x, y, z, t) \frac{C_{00}^{\gamma-1}(x, y, z, t)}{P^{\gamma}(x, y, z, T)} \frac{\partial C_{00}(x, y, z, t)}{\partial z}\right]\right\} D_{0 L}+ \\
+D_{0 L}\left\{\frac{\partial}{\partial x}\left[\frac{C_{00}^{\gamma}(x, y, z, t)}{P^{\gamma}(x, y, z, T)} \frac{\partial C_{10}(x, y, z, t)}{\partial x}\right]+\frac{\partial}{\partial y}\left[\frac{C_{00}^{\gamma}(x, y, z, t)}{P^{\gamma}(x, y, z, T)} \frac{\partial C_{10}(x, y, z, t)}{\partial y}\right]+\right. \\
\left.+\frac{\partial}{\partial z}\left[\frac{C_{00}^{\gamma}(x, y, z, t)}{P^{\gamma}(x, y, z, T)} \frac{\partial C_{10}(x, y, z, t)}{\partial z}\right]\right\}+D_{0 L}\left\{\frac{\partial}{\partial x}\left[g_{L}(x, y, z, T) \frac{\partial C_{01}(x, y, z, t)}{\partial x}\right]+\right. \\
\left.+\frac{\partial}{\partial y}\left[g_{L}(x, y, z, T) \frac{\partial C_{01}(x, y, z, t)}{\partial y}\right]+\frac{\partial}{\partial z}\left[C_{L}(x, y, z, T) \frac{\partial C_{01}(x, y, z, t)}{\partial z}\right]\right\} ; \\
\left.\frac{\partial C_{i j}(x, y, z, t)}{\partial x}\right|_{x=0}=0,\left.\frac{\partial C_{i j}(x, y, z, t)}{\partial x}\right|_{x=L_{x}}=0,\left.\frac{\partial C_{i j}(x, y, z, t)}{\partial y}\right|_{y=0}=0,\left.\frac{\partial C_{i j}(x, y, z, t)}{\partial y}\right|_{y=L_{y}}=0 \\
=
\end{gathered}
$$

Solutions of the above equations with account boundary and initial have been calculated by Fourier approach. The result of calculation could be written as

$$
C_{00}(x, y, z, t)=\frac{1}{L_{x} L_{y} L_{z}}+\frac{2}{L_{x} L_{y} L_{z}} \sum_{n=1}^{\infty} F_{n C} c_{n}(x) c_{n}(y) c_{n}(z) e_{n C}(t),
$$

where $e_{n C}(t)=\exp \left[-\pi^{2} n^{2} D_{0 C} t\left(\frac{1}{L_{x}^{2}}+\frac{1}{L_{y}^{2}}+\frac{1}{L_{z}^{2}}\right)\right], F_{n C}=\int_{0}^{L_{x}} c_{n}(u) \int_{0}^{L_{y}} c_{n}(v) \int_{0}^{L_{z}} f_{C}(u, v, w) c_{n}(w) d w d v d u$;

$$
\begin{aligned}
& C_{i 0}(x, y, z, t)=-\frac{2 \pi}{L_{x}^{2} L_{y} L_{z}} \sum_{n=1}^{\infty} n F_{n C} c_{n}(x) c_{n}(y) c_{n}(z) e_{n C}(t) \int_{0}^{t} e_{n C}(-\tau) \int_{0}^{L_{x}} s_{n}(u) \int_{0}^{L_{y}} c_{n}(v) \int_{0}^{L_{z}} g_{L}(u, v, w, T) \times \\
& \times c_{n}(w) \frac{\partial C_{i-10}(u, v, w, \tau)}{\partial u} d w d v d u d \tau-\frac{2 \pi}{L_{x} L_{y}^{2} L_{z}} \sum_{n=1}^{\infty} n F_{n C} c_{n}(x) c_{n}(y) c_{n}(z) e_{n C}(t) \int_{0}^{t} e_{n C}(-\tau) \times
\end{aligned}
$$


International Journal on Organic Electronics (IJOE) Vol.6, No.2, April 2017

$$
\begin{aligned}
& \times \int_{0}^{L_{x}} c_{n}(u) \int_{0}^{L_{y}} s_{n}(v) \int_{0}^{L_{z}} c_{n}(v) g_{L}(u, v, w, T) \frac{\partial C_{i-10}(u, v, w, \tau)}{\partial v} d w d v d u d \tau-\frac{2 \pi}{L_{x} L_{y} L_{z}^{2}} \sum_{n=1}^{\infty} n F_{n C} e_{n C}(t) \times \\
& \times c_{n}(x) c_{n}(y) c_{n}(z) \int_{0}^{t} e_{n C}(-\tau) \int_{0}^{L_{x}} c_{n}(u) \int_{0}^{L_{y}} c_{n}(v) \int_{0}^{L_{z}} s_{n}(v) g_{L}(u, v, w, T) \frac{\partial C_{i-10}(u, v, w, \tau)}{\partial w} d w d v d u d \tau, i \geq 1 ; \\
& C_{01}(x, y, z, t)=-\frac{2 \pi}{L_{x}^{2} L_{y} L_{z}} \sum_{n=1}^{\infty} n F_{n C} c_{n}(x) c_{n}(y) c_{n}(z) e_{n C}(t) \int_{0}^{t} e_{n C}(-\tau) \int_{0}^{L_{x}} s_{n}(u) \int_{0}^{L_{y}} c_{n}(v) \int_{0}^{L_{z}} c_{n}(w) \times \\
& \times \frac{C_{00}^{\gamma}(u, v, w, \tau)}{P^{\gamma}(u, v, w, T)} \frac{\partial C_{00}(u, v, w, \tau)}{\partial u} d w d v d u d \tau-\frac{2 \pi}{L_{x} L_{y}^{2} L_{z}} \sum_{n=1}^{\infty} n F_{n C} c_{n}(x) c_{n}(y) c_{n}(z) e_{n C}(t) \times \\
& \times \int_{0}^{t} e_{n C}(-\tau) \int_{0}^{L_{x}} c_{n}(u) \int_{0}^{L_{y}} s_{n}(v) \int_{0}^{L_{z}} c_{n}(w) \frac{C_{00}^{\gamma}(u, v, w, \tau)}{P^{\gamma}(u, v, w, T)} \frac{\partial C_{00}(u, v, w, \tau)}{\partial v} d w d v d u d \tau-\frac{2 \pi}{L_{x} L_{y} L_{z}^{2}} \sum_{n=1}^{\infty} n e_{n C}(t) \times \\
& \times F_{n C} c_{n}(x) c_{n}(y) c_{n}(z) \int_{0}^{t} e_{n C}(-\tau) \int_{0}^{L_{x}} c_{n}(u) \int_{0}^{L_{y}} c_{n}(v) \int_{0}^{L_{z}} s_{n}(w) \frac{C_{00}^{\gamma}(u, v, w, \tau)}{P^{\gamma}(u, v, w, T)} \frac{\partial C_{00}(u, v, w, \tau)}{\partial w} d w d v d u d \tau ; \\
& C_{02}(x, y, z, t)=-\frac{2 \pi}{L_{x}^{2} L_{y} L_{z}} \sum_{n=1}^{\infty} n F_{n C} c_{n}(x) c_{n}(y) c_{n}(z) e_{n C}(t) \int_{0}^{t} e_{n C}(-\tau) \int_{0}^{L_{x}} s_{n}(u) \int_{0}^{L_{y}} c_{n}(v) \int_{0}^{L_{\tilde{z}}} c_{n}(w) \times \\
& \times C_{01}(u, v, w, \tau) \frac{C_{00}^{\gamma-1}(u, v, w, \tau)}{P^{\gamma}(u, v, w, T)} \frac{\partial C_{00}(u, v, w, \tau)}{\partial u} d w d v d u d \tau-\frac{2 \pi}{L_{x} L_{v}^{2} L_{z}} \sum_{n=1}^{\infty} F_{n C} c_{n}(x) c_{n}(y) \times \\
& \times n c_{n}(z) e_{n C}(t) \int_{0}^{t} e_{n C}(-\tau) \int_{0}^{L_{x}} c_{n}(u) \int_{0}^{L_{y}} s_{n}(v) \int_{0}^{L_{z}} C_{01}(u, v, w, \tau) \frac{C_{00}^{\gamma-1}(u, v, w, \tau)}{P^{\gamma}(u, v, w, T)} \frac{\partial C_{00}(u, v, w, \tau)}{\partial v} \times \\
& \times c_{n}(w) d w d v d u d \tau-\frac{2 \pi}{L_{x} L_{y} L_{z}^{2}} \sum_{n=1}^{\infty} n F_{n C} c_{n}(x) c_{n}(y) c_{n}(z) e_{n C}(t) \int_{0}^{t} e_{n C}(-\tau) \int_{0}^{L_{x}} c_{n}(u) \int_{0}^{L_{y}} c_{n}(v) \times \\
& \times \int_{0}^{L_{z}} s_{n}(w) C_{01}(u, v, w, \tau) \frac{C_{00}^{\gamma-1}(u, v, w, \tau)}{P^{\gamma}(u, v, w, T)} \frac{\partial C_{00}(u, v, w, \tau)}{\partial w} d w d v d u d \tau-\frac{2 \pi}{L_{x}^{2} L_{y} L_{z}} \sum_{n=1}^{\infty} n c_{n}(x) \times \\
& \times F_{n C} c_{n}(y) c_{n}(z) e_{n C}(t) \int_{0}^{t} e_{n C}(-\tau) \int_{0}^{L_{x}} s_{n}(u) \int_{0}^{L_{y}} c_{n}(v) \int_{0}^{L_{z}} c_{n}(w) C_{01}(u, v, w, \tau) \frac{\partial C_{00}(u, v, w, \tau)}{\partial u} \times \\
& \times \frac{C_{00}^{\gamma-1}(u, v, w, \tau)}{P^{\gamma}(u, v, w, T)} d w d v d u d \tau-\frac{2 \pi}{L_{x} L_{y}^{2} L_{z}} \sum_{n=1}^{\infty} n F_{n C} c_{n}(x) c_{n}(y) c_{n}(z) e_{n C}(t) \int_{0}^{t} e_{n C}(-\tau) \int_{0}^{L_{x}} c_{n}(u) \times \\
& \times \int_{0}^{L_{y}} S_{n}(v) \int_{0}^{L_{z}} c_{n}(w) C_{01}(u, v, w, \tau) \frac{C_{00}^{\gamma-1}(u, v, w, \tau)}{P^{\gamma}(u, v, w, T)} \frac{\partial C_{00}(u, v, w, \tau)}{\partial v} d w d v d u d \tau-\frac{2 \pi}{L_{x} L_{y} L_{z}^{2}} \sum_{n=1}^{\infty} n \times \\
& \times F_{n C} c_{n}(x) c_{n}(y) c_{n}(z) e_{n C}(t) \int_{0}^{t} e_{n C}(-\tau) \int_{0}^{L_{x}} c_{n}(u) \int_{0}^{L_{y}} c_{n}(v) \int_{0}^{L_{\tilde{z}}} s_{n}(w) C_{01}(u, v, w, \tau) \frac{C_{00}^{\gamma-1}(u, v, w, \tau)}{P^{\gamma}(u, v, w, T)} \times \\
& \times \frac{\partial C_{00}(u, v, w, \tau)}{\partial w} d w d v d u d \tau-\frac{2 \pi}{L_{x}^{2} L_{y} L_{z}} \sum_{n=1}^{\infty} F_{n C} c_{n}(x) c_{n}(y) c_{n}(z) e_{n C}(t) \int_{0}^{t} e_{n C}(-\tau) \int_{0}^{L_{x}} s_{n}(u) \times \\
& \times n \int_{0}^{L_{y}} c_{n}(v) \int_{0}^{L_{z}} c_{n}(w) \frac{C_{00}^{\gamma}(u, v, w, \tau)}{P^{\gamma}(u, v, w, T)} \frac{\partial C_{01}(u, v, w, \tau)}{\partial u} d w d v d u d \tau-\frac{2 \pi}{L_{x} L_{y}^{2} L_{z}} \sum_{n=1}^{\infty} c_{n}(x) e_{n C}(t) \times \\
& \times F_{n C} c_{n}(y) \int_{0}^{t} e_{n C}(-\tau) \int_{0}^{L_{x}} c_{n}(u) \int_{0}^{L_{y}} s_{n}(v) \int_{0}^{L_{z}} c_{n}(w) \frac{C_{00}^{\gamma}(u, v, w, \tau)}{P^{\gamma}(u, v, w, T)} \frac{\partial C_{01}(u, v, w, \tau)}{\partial v} d w d v d u d \tau \times \\
& \times n c_{n}(z)-\frac{2 \pi}{L_{x} L_{y} L_{z}^{2}} \sum_{n=1}^{\infty} n F_{n C} c_{n}(x) c_{n}(y) c_{n}(z) e_{n C}(t) \int_{0}^{t} e_{n C}(-\tau) \int_{0}^{L_{x}} c_{n}(u) \int_{0}^{L_{y}} c_{n}(v) \int_{0}^{L_{z}} s_{n}(w) \times
\end{aligned}
$$


International Journal on Organic Electronics (IJOE) Vol.6, No.2, April 2017

$$
\begin{aligned}
& \times \frac{C_{00}^{\gamma}(u, v, w, \tau)}{P^{\gamma}(u, v, w, T)} \frac{\partial C_{01}(u, v, w, \tau)}{\partial w} d w d v d u d \tau \\
& C_{11}(x, y, z, t)=-\frac{2 \pi}{L_{x}^{2} L_{y} L_{z}} \sum_{n=1}^{\infty} n F_{n C} c_{n}(x) c_{n}(y) c_{n}(z) e_{n C}(t) \int_{0}^{t} e_{n C}(-\tau) \int_{0}^{L_{x}} s_{n}(u) \int_{0}^{L_{y}} c_{n}(v) \int_{0}^{L_{z}} c_{n}(w) \times \\
& \times g_{L}(u, v, w, T) \frac{\partial C_{01}(u, v, w, \tau)}{\partial u} d w d v d u d \tau-\frac{2 \pi}{L_{x} L_{y}^{2} L_{z}} \sum_{n=1}^{\infty} n F_{n C} c_{n}(x) c_{n}(y) c_{n}(z) e_{n C}(t) \times \\
& \times \int_{0}^{t} e_{n C}(-\tau) \int_{0}^{L_{x}} c_{n}(u) \int_{0}^{L_{y}} s_{n}(v) \int_{0}^{L_{z}} c_{n}(w) g_{L}(u, v, w, T) \frac{\partial C_{01}(u, v, w, \tau)}{\partial v} d w d v d u d \tau-\frac{2 \pi}{L_{x} L_{y} L_{z}^{2}} \times \\
& \times \sum_{n=1}^{\infty} n e_{n C}(t) \int_{0}^{t} e_{n C}(-\tau) \int_{0}^{L_{x}} c_{n}(u) \int_{0}^{L_{y}} c_{n}(v) \int_{0}^{L_{z}} s_{n}(w) g_{L}(u, v, w, T) \frac{\partial C_{01}(u, v, w, \tau)}{\partial w} d w d v d u d \tau \times \\
& \times F_{n C} c_{n}(x) c_{n}(y) c_{n}(z)-\frac{2 \pi}{L_{x}^{2} L_{y} L_{z}} \sum_{n=1}^{\infty} F_{n C} c_{n}(x) c_{n}(y) c_{n}(z) e_{n C}(t) \int_{0}^{t} e_{n C}(-\tau) \int_{0}^{L_{x}} s_{n}(u) \int_{0}^{L_{y}} c_{n}(v) \times \\
& \times n \int_{0}^{L_{z}} c_{n}(w) \frac{C_{00}^{\gamma}(u, v, w, \tau)}{P^{\gamma}(u, v, w, T)} \frac{\partial C_{10}(u, v, w, \tau)}{\partial u} d w d v d u d \tau-\frac{2 \pi}{L_{x} L_{y}^{2} L_{z}} \sum_{n=1}^{\infty} n F_{n C} c_{n}(x) c_{n}(y) \times \\
& \times c_{n}(z) e_{n C}(t) \int_{0}^{t} e_{n C}(-\tau) \int_{0}^{L_{x}} c_{n}(u) \int_{0}^{L_{y}} s_{n}(v) \int_{0}^{L_{z}} c_{n}(w) \frac{C_{00}^{\gamma}(u, v, w, \tau)}{P^{\gamma}(u, v, w, T)} \frac{\partial C_{10}(u, v, w, \tau)}{\partial v} d w d v d u d \tau- \\
& -\frac{2 \pi}{L_{x} L_{y} L_{z}^{2}} \sum_{n=1}^{\infty} n F_{n C} c_{n}(x) c_{n}(y) c_{n}(z) e_{n C}(t) \int_{0}^{t} e_{n C}(-\tau) \int_{0}^{L_{x}} c_{n}(u) \int_{0}^{L_{y}} c_{n}(v) \int_{0}^{L_{z}} s_{n}(w) \frac{C_{00}^{\gamma}(u, v, w, \tau)}{P^{\gamma}(u, v, w, T)} \times \\
& \times \frac{\partial C_{10}(u, v, w, \tau)}{\partial w} d w d v d u d \tau-\frac{2 \pi}{L_{x}^{2} L_{y} L_{z}} \sum_{n=1}^{\infty} n F_{n C} c_{n}(x) c_{n}(y) c_{n}(z) e_{n C}(t) \int_{0}^{t} e_{n C}(-\tau) \int_{0}^{L_{x}} s_{n}(u) \times \\
& \times \int_{0}^{L_{y}} c_{n}(v) \int_{0}^{L_{z}} c_{n}(w) C_{10}(u, v, w, \tau) \frac{C_{00}^{\gamma-1}(u, v, w, \tau)}{P^{\gamma}(u, v, w, T)} \frac{\partial C_{00}(u, v, w, \tau)}{\partial u} d w d v d u d \tau-\frac{2 \pi}{L_{x} L_{y}^{2} L_{z}} \sum_{n=1}^{\infty} n \times \\
& \times F_{n C} c_{n}(x) c_{n}(y) c_{n}(z) e_{n C}(t) \int_{0}^{t} e_{n C}(-\tau) \int_{0}^{L_{x}} c_{n}(u) \int_{0}^{L_{y}} s_{n}(v) \int_{0}^{L_{z}} c_{n}(w) \frac{C_{00}^{\gamma-1}(u, v, w, \tau)}{P^{\gamma}(u, v, w, T)} \frac{\partial C_{00}(u, v, w, \tau)}{\partial v} \times \\
& \times C_{10}(u, v, w, \tau) d w d v d u d \tau-\frac{2 \pi}{L_{x} L_{y} L_{z}^{2}} \sum_{n=1}^{\infty} n F_{n C} c_{n}(x) c_{n}(y) c_{n}(z) e_{n C}(t) \int_{0}^{t} e_{n C}(-\tau) \int_{0}^{L_{x}} c_{n}(u) \times \\
& \times \int_{0}^{L_{y}} c_{n}(v) \int_{0}^{L_{z}} s_{n}(w) C_{10}(u, v, w, \tau) \frac{C_{00}^{\gamma-1}(u, v, w, \tau)}{P^{\gamma}(u, v, w, T)} \frac{\partial C_{00}(u, v, w, \tau)}{\partial w} d w d v d u d \tau .
\end{aligned}
$$

